\title{
A basic thin shell triangle with only translational DOFs for large strain plasticity
}

\author{
Fernando G. Flores ${ }^{1}$ and Eugenio Oñate ${ }^{2, *, \dagger}$ \\ ${ }^{1}$ National University of Cordoba, Casilla de Correo 916, 5000 Córdoba, Argentina \\ ${ }^{2}$ International Center for Numerical Methods in Engineering, Edificio C1, Gran Capitán s/n, \\ 08034 Barcelona, Spain
}

\begin{abstract}
SUMMARY
A simple finite element triangle for thin shell analysis is presented. It has only nine translational degrees of freedom and is based on a total Lagrangian formulation. Large strain plasticity is considered using a logarithmic strain-stress pair. A plane stress isotropic behaviour with an additive decomposition of elastic and plastic strains is assumed. A hyperelastic law is considered for the elastic part while for the plastic part a von Mises yield function with non-linear isotropic hardening is adopted. The element is an extension of a previous similar rotation-free triangle element based upon an updated Lagrangian formulation with hypoelastic constitutive law. The element termed BST (for basic shell triangle) has been implemented in an explicit (hydro-) code adequate to simulate sheet-stamping processes and in an implicit static/dynamic code. Several examples are shown to assess the performance of the present formulation. Copyright (c) 2001 John Wiley \& Sons, Ltd.
\end{abstract}

KEY WORDS: triangular finite elements; thin shell; rotation-free; large strain plasticity

\section{INTRODUCTION}

Several authors have tried to derive triangular plate and shell finite elements with displacements as the only nodal variables [1-6]. In essence, all methods attempt to approximate the curvatures over an element in terms of the deflection of the nodes in a surrounding patch of elements. Applications of these displacement-based shell elements to sheet forming analysis using explicit dynamic codes were reported by Yang et al. [7], Brunet and Sabourin [8], and Rio et al. [9].

Oñate and co-workers have derived rotation-free thin (Kirchhoff-Love) plate and shell triangles from a slightly different perspective. The basic idea is to combine the standard finite

\footnotetext{
*Correspondence to: Eugenio Oñate, International Center for Numerical Methods in Engineering, Edificio C1, Campus Norte UPC, Gran Capitan, s/n 08034, Barcelona, Spain

†E-mail: onate@cimne.upc.es

Contact/grant sponsor: CONICET

Contact/grant sponsor: CONICOR
}

Copyright (c) 2001 John Wiley \& Sons, Ltd.

Received 6 January 2000

Revised 7 July 2000 
element (FE) interpolation with finite volume (FV) concepts [10-12]. This allows to express the curvatures over a control domain in terms of the displacement gradients along the domain edges. These gradients are in turn written in terms of the deflections of the nodes belonging to an element patch surrounding the control domain and this leads to a relationship between curvatures and nodal displacements [14]. In Reference [13], it is also shown how a mixed $\mathrm{Hu}$-Washizu functional provides an adequate framework for the derivation of the discretized equations for the new rotation-free plate and shell triangles combining FE and FV approaches.

The above ideas were used in References $[14,13]$ to derive a simple rotation-free threenode shell triangle termed BST (for basic shell triangle) showing an excellent behaviour for linear analysis of plate and shell structures. An extension of the BST element to the nonlinear analysis of shells was implemented in an explicit dynamic code using an Updated

Lagrangian Formulation and a hypo-elastic constitutive model. Excellent numerical results were obtained related mainly to sheet stamping problems and were reported in References [15-17]. The behaviour of the BST element in an implicit code has some drawbacks related to its lack of objectivity due to the hypo-elastic constitutive law and to the way in which the deformations are integrated in time, demanding short steps (as in an explicit code) for an accurate modelling.

A large strain formulation for the BST element using a total Lagrangian description is presented in this paper. The constitutive model is based on von-Mises plasticity with nonlinear isotropic hardening formulated in terms of Hencky stresses. The algorithmic elastoplastic tangent matrix relating IItencky's stress and strain increments is transformed to the two-dimensional Piola-Kirchhoff stress and Green-Lagrange strain spaces. The content of the paper is the following. Some basic aspedts of the geometric definition of the element are given in Section 2. Next, the computation of the membrane strains and the curyatures is presented. Here the concept of 'patch of elements' is again essential to express the element curvatures terms of the displacement of the patch nodes alone. In Sections 6 and 7 , the derivation of the residual force vector and the tangent stiffness matrix is explained in some detail. Details of

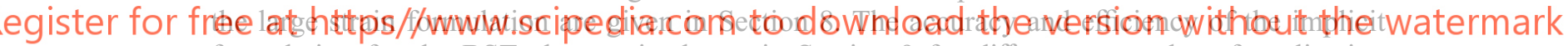
formulation for the BST element is shown in Section 9 for different examples of application to non-linear shell analysis and the simulation of sheet stamping problems.

\section{DEFINITION OF THE GEOMETRY}

The element has only three vertex nodes with three degrees of freedom (DOF) at each node. This leads to a standard constant strain triangle for the membrane behaviour. For the bending behaviour three DOFs are not enough, so a cell-element (the control domain) is defined by the main element and the three adjacent elements (see Figure 2).

The node-ordering in the cell-element is as follows:

- The nodes in the main element (M) are numbered locally as 1,2 and 3. They are defined counterclockwise around the positive normal.

- The sides in the main element are numbered locally as 1,2 , and 3 . They are defined by the local node opposite to the side.

- The adjacent elements (which are part of the cell) are numbered with the number associated to the common side. 


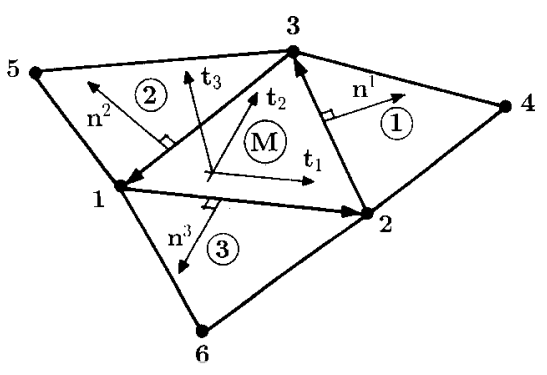

Figure 1. Element patch definition.

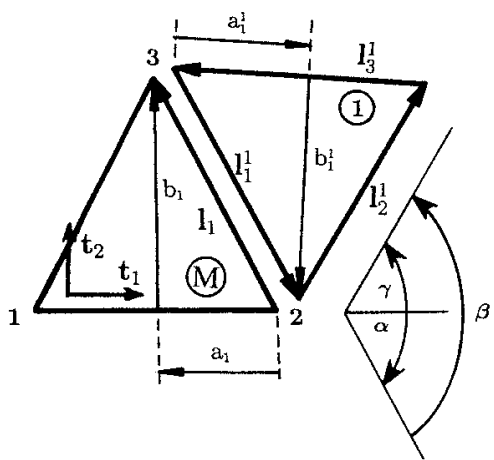

Figure 2. Angles for the definition of the local system.

- The extra nodes of the cell are numbered locally as 4,5 and 6, corresponding to nodes on adjacent elements opposite to sides 1,2 and 3, respectively.

- The connectivities in the adjacent element are defined beginning with the extra node. This is done as follows:
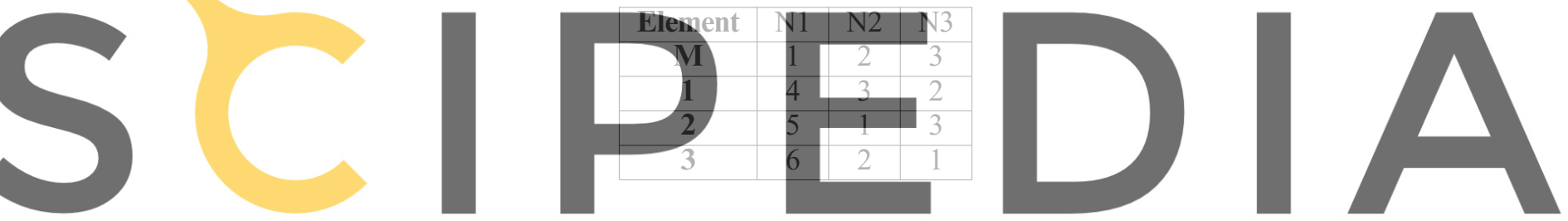

A local co-ordinate system must be defined for the patch. Among the different possibilities,

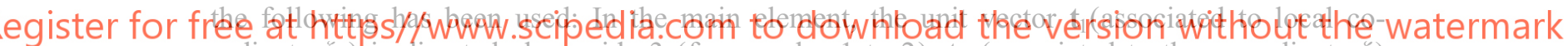
ordinate $\xi_{1}$ ) is directed along side 3 (from nodes 1 to 2 ), $t_{3}$ (associated to the co-ordinate $\zeta$ ) is the unit normal to the plane, and finally $\mathrm{t}_{2}=\mathrm{t}_{3} \times \mathrm{t}_{1}$ (associated to the co-ordinate $\xi_{2}$ ). One can then define for each element:

$$
\begin{aligned}
\mathbf{l}_{I} & =e_{I J} \boldsymbol{\varphi}^{J(0)} \\
e_{I J} & =\left[\begin{array}{ccc}
0 & -1 & 1 \\
1 & 0 & -1 \\
-1 & 1 & 0
\end{array}\right] \\
a_{I} & =\mathbf{l}_{I} \cdot \mathbf{t}_{1} \\
b_{I} & =\mathbf{l}_{I} \cdot \mathbf{t}_{2} \\
2 A^{(0)} & =a_{1} b_{2}-a_{2} b_{1}
\end{aligned}
$$

where $\boldsymbol{\varphi}^{I(0)}$ are the original nodal co-ordinates, $\mathbf{l}_{I}$ are the oriented side vectors, $\left(a_{I}, b_{I}\right)$ are their projections over the local directions $\mathbf{t}_{1}$ and $\mathbf{t}_{2}$, and $A$ is the triangle area. An upper index 0 in brackets denotes values for the original (undeformed) configuration. 
In the adjacent elements, the local system is chosen to match the local system in the main element. For an initially flat surface this co-ordinate system is easily continued over the rest of the triangles in the patch. For an initially curved surface some care must be taken as shown next.

The projections of the first side of each adjacent element ' $J$ ' (sharing side with the main triangle) are simply

$$
\begin{aligned}
& a_{1}^{J}=-a_{J} \\
& b_{1}^{J}=-b_{J}
\end{aligned}
$$

Note that with an upper index we are denoting geometric parameters associated to the adjacent elements to the main element $M$ (see Figure 2).

We now define $\alpha$ as the angle between side 1 of the adjacent element with local axis 1 (see Figure 2). Then

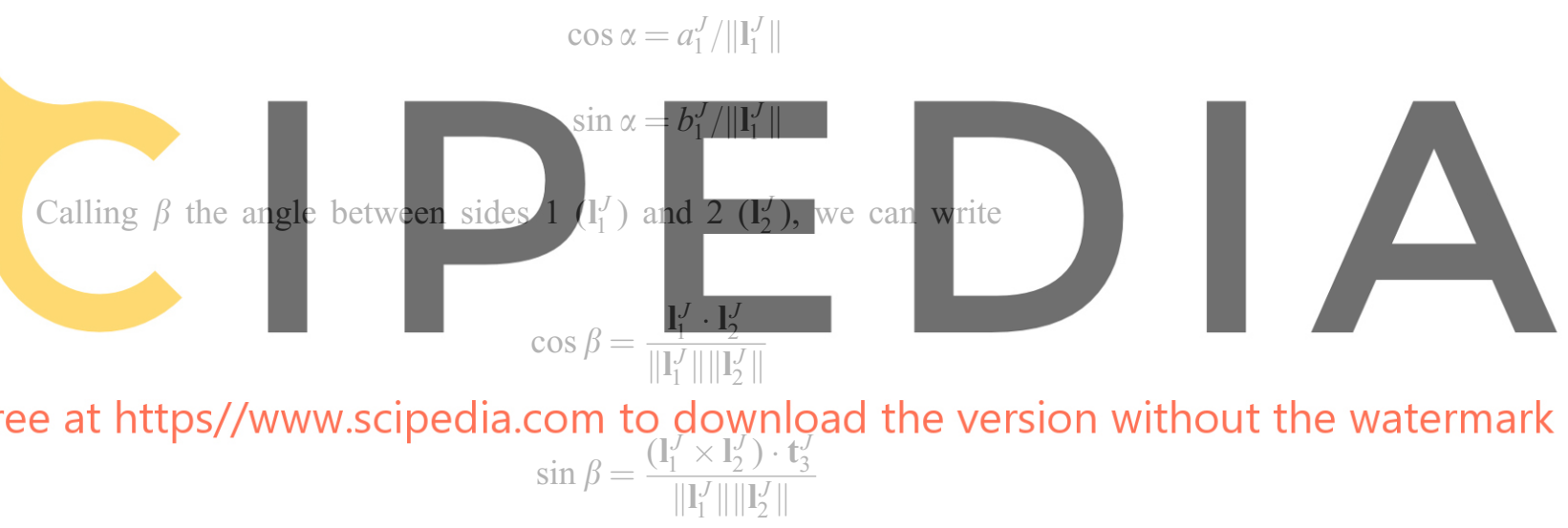

where $\mathbf{t}_{3}^{J}$ is the unit normal vector to the adjacent element $J$. These two angles allow to compute $\gamma$, the angle between side 2 of the adjacent element and the local axis 1 , as the sum of angles $\alpha$ and $\beta$. Finally, the side projections are computed as

$$
\begin{aligned}
& a_{2}^{J}=\left\|\mathbf{I}_{2}^{J}\right\| \cos \gamma \\
& b_{2}^{J}=\left\|\mathbf{I}_{2}^{J}\right\| \sin \gamma \\
& a_{3}^{J}=-a_{1}^{J}-a_{2}^{J} \\
& b_{3}^{J}=-b_{1}^{J}-b_{2}^{J}
\end{aligned}
$$

with $\gamma=\alpha+\beta$. 
The second equality assumes that the deformation is isochoric (even elastic deformation). For metal sheets, where elastic deformations are small and plastic flow is taken as isochoric, this approximation has no practical consequences. For materials with other characteristics this assumption must be reviewed.

The assumption that fibres originally normal to the surface in the reference configuration are also normal to the surface in the current configuration (Kirchhoff hypothesis) is adopted.

The changes of curvatures are computed as the difference between the values in the current and original configurations

$$
\chi_{\alpha \beta}=k_{\alpha \beta}-k_{\alpha \beta}^{(0)}=-\mathbf{t}_{3} \cdot \boldsymbol{\varphi}^{\prime} \beta \alpha+\mathbf{t}_{3}^{(0)} \cdot \boldsymbol{\varphi}^{(0)}{ }_{\beta \alpha}
$$

As in the original BST element [14], the curvatures are assumed to be constant within each element. For this purpose, an averaging of the curvatures $k_{\alpha, \beta}$ is made over the element in a mean integral sense as

$$
k_{\alpha \beta}=\frac{-1}{A^{(0)}} \int_{A^{(0)}} \mathbf{t}_{3} \cdot \boldsymbol{\varphi}^{\prime} \beta \alpha \mathrm{d} A^{(0)}
$$

Integrating by parts the right-hand side of Equation (12) gives
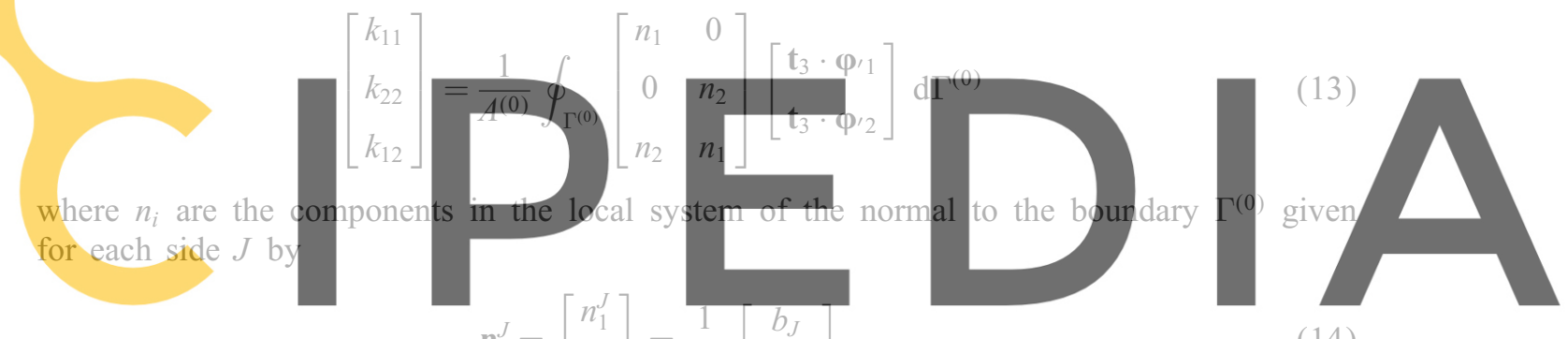

(14)

egister for free at https//www.scipedia.con to download the version without the watermark

Equation (13) can be found to be equivalent to standard conservation laws used in finite volume procedures as described in Reference [12] (see also remarks in Reference [14]).

Due to the definition of $\mathbf{t}_{3}$, then $\mathbf{t}_{3} \cdot \boldsymbol{\varphi}^{\prime} \alpha=0$, i.e. the curvature of a flat triangle is zero. In order to compute the line integral of Equation (13), the following averaging procedure is used: along each side of the triangle the average value of $\varphi^{\prime} \alpha$ between the main triangle and the adjacent one is taken leading to

$$
\left[\begin{array}{l}
k_{11} \\
k_{22} \\
k_{12}
\end{array}\right]=\frac{1}{A^{(0)}} \sum_{I=1}^{3}\left[\begin{array}{cc}
-b_{I} & 0 \\
0 & a_{I} \\
a_{I} & -b_{I}
\end{array}\right]\left[\begin{array}{l}
\mathbf{t}_{3} \cdot \frac{1}{2}\left(\boldsymbol{\varphi}_{\prime 1}^{\mathrm{M}}+\boldsymbol{\varphi}_{\prime 1}^{I}\right) \\
\mathbf{t}_{3} \cdot \frac{1}{2}\left(\boldsymbol{\varphi}_{\prime 2}^{\mathrm{M}}+\boldsymbol{\varphi}_{/ 2}^{I}\right)
\end{array}\right]
$$

Recalling that $\sum a_{I}=\sum b_{I}=0$ and also due to the condition $\mathbf{t}_{3} \cdot \boldsymbol{\varphi}_{{ }^{\prime}}^{\mathrm{M}}=0$ in the main triangle one has

$$
\left[\begin{array}{l}
k_{11} \\
k_{22} \\
k_{12}
\end{array}\right]=\frac{1}{2 A^{(0)}} \sum_{I=1}^{3}\left[\begin{array}{cc}
-b_{I} & 0 \\
0 & a_{I} \\
a_{I} & -b_{I}
\end{array}\right]\left[\begin{array}{l}
\mathbf{t}_{3} \cdot \boldsymbol{\varphi}_{\prime 1}^{I} \\
\mathbf{t}_{3} \cdot \boldsymbol{\varphi}_{\prime 2}^{I}
\end{array}\right]
$$




$$
=\sum_{I=1}^{3}\left[\begin{array}{cc}
N_{\prime 1}^{I} & 0 \\
0 & N_{\prime 2}^{I} \\
N_{\prime 2}^{I} & N_{\prime 1}^{I}
\end{array}\right]\left[\begin{array}{l}
\mathbf{t}_{3} \cdot \boldsymbol{\varphi}_{\prime 1}^{I} \\
\mathbf{t}_{3} \cdot \boldsymbol{\varphi}_{\prime 2}^{I}
\end{array}\right]
$$

This can be seen as the projection of the local derivatives in the adjacent triangles $\boldsymbol{\varphi}_{{ }_{\alpha}}^{I}$ (denoting with an index $I$ values associated to the adjacent element) over the normal to the main triangle $\mathbf{t}_{3}$. As the triangles have a common side $\left(\mathbf{t}_{3} \cdot \boldsymbol{\varphi}_{s_{s}}^{I}=0\right)$, with $\boldsymbol{\varphi}_{s_{s}}^{I}$ being the derivative along the side, only the derivative along the side normal $\left(\boldsymbol{\varphi}_{\prime_{n}}^{I}\right)$ has non-zero component over $\mathbf{t}_{3}$, leading to

$$
\left[\begin{array}{l}
\mathbf{t}_{3} \cdot \boldsymbol{\varphi}_{\prime 1}^{I} \\
\mathbf{t}_{3} \cdot \boldsymbol{\varphi}_{\prime 2}^{I}
\end{array}\right]=\mathbf{n}^{I}\left(\mathbf{t}_{3} \cdot \boldsymbol{\varphi}_{\prime n}^{I}\right)=\frac{1}{\left|\mathbf{I}_{I}\right|}\left[\begin{array}{c}
b_{I} \\
-a_{I}
\end{array}\right]\left(\mathbf{t}_{3} \cdot \boldsymbol{\varphi}_{\prime n}^{I}\right)
$$

\section{These projections will be denoted as}

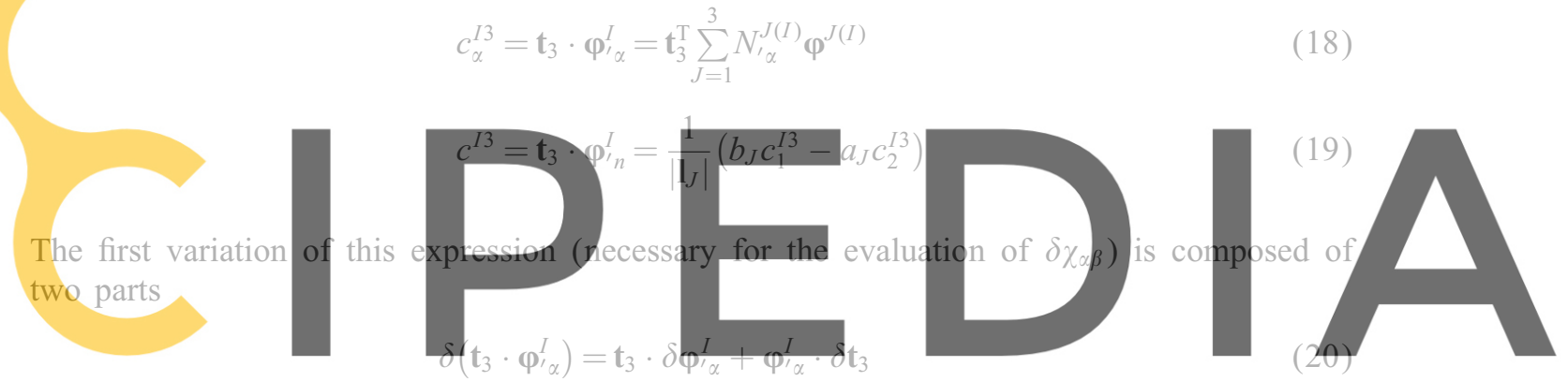

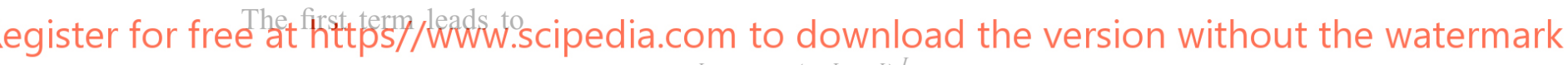

$$
\mathbb{t}_{3} \cdot \delta \varphi^{I} \alpha=\mathbb{t}_{3} \cdot\left(N_{i \alpha}^{J} \delta \mathbf{u}^{J}\right)^{I}
$$

For the second term, recalling Equation (9), one has

$$
\begin{aligned}
& \delta \mathbf{t}_{3}=\lambda\left(\delta \boldsymbol{\varphi}_{\prime_{1}} \times \boldsymbol{\varphi}_{\prime 2}+\boldsymbol{\varphi}_{\prime_{1}} \times \delta \boldsymbol{\varphi}^{\prime 2}\right)+\delta \lambda\left(\boldsymbol{\varphi}_{\prime_{1}} \times \boldsymbol{\varphi}_{\prime 2}\right) \\
& =\lambda\left(\delta \boldsymbol{\varphi}^{\prime 1} \times \boldsymbol{\varphi}_{22}+\boldsymbol{\varphi}^{\prime} 1 \times \delta \boldsymbol{\varphi}_{\prime 2}\right)+\frac{\delta \lambda}{\lambda} \mathbf{t}_{3} \\
& =\lambda\left(-\hat{\boldsymbol{\varphi}}_{22} \delta \boldsymbol{\varphi}^{\prime} 1+\hat{\boldsymbol{\varphi}}_{1} \delta \boldsymbol{\varphi}_{2}\right)+\frac{\delta \lambda}{\lambda} \mathbf{t}_{3}
\end{aligned}
$$

where $\hat{\boldsymbol{\varphi}}_{\prime_{\alpha}}$ is the corresponding skew-symmetric matrix associated to vector $\boldsymbol{\varphi}^{\prime} \alpha$. Noting that $\mathbf{t}_{3} \cdot \delta \mathbf{t}_{3}=0$ and resolving $\delta \mathbf{t}_{3}$ in components $\left(\delta t_{3 \alpha}\right)$ on the convective base $\boldsymbol{\varphi}^{\prime \alpha}$

$$
\begin{aligned}
& \delta t_{31}=\boldsymbol{\varphi}_{\prime_{1}} \cdot \delta \mathbf{t}_{3}=\lambda\left(-\boldsymbol{\varphi}_{\prime_{1}} \cdot \hat{\boldsymbol{\varphi}}_{\prime_{2}} \delta \boldsymbol{\varphi}^{\prime} 1+\boldsymbol{\varphi}_{\prime 1} \cdot \hat{\boldsymbol{\varphi}}_{\prime_{1}} \delta \boldsymbol{\varphi}^{\prime 2}\right)=\lambda\left(-\boldsymbol{\varphi}_{\prime_{1}} \times \boldsymbol{\varphi}_{22} \delta \boldsymbol{\varphi}_{\prime 1}\right)=-\mathbf{t}_{3} \cdot \delta \boldsymbol{\varphi}^{\prime} 1 \\
& \delta t_{32}=\boldsymbol{\varphi}^{\prime 2} \cdot \delta \mathbf{t}_{3}=\lambda\left(-\boldsymbol{\varphi}_{22} \cdot \hat{\boldsymbol{\varphi}}_{{ }_{2}} \delta \boldsymbol{\varphi}_{\prime 1}+\boldsymbol{\varphi}_{22} \cdot \hat{\boldsymbol{\varphi}}_{{ }_{1}} \delta \boldsymbol{\varphi}^{\prime 2}\right)=\lambda\left(\boldsymbol{\varphi}_{22} \times \boldsymbol{\varphi}_{\prime 1} \delta \boldsymbol{\varphi}^{\prime 2}\right)=-\mathbf{t}_{3} \cdot \delta \boldsymbol{\varphi}^{\prime 2}
\end{aligned}
$$


which leads to

$$
\delta \mathbf{t}_{3}=\delta t_{31} \tilde{\boldsymbol{\varphi}}_{1}+\delta t_{32} \tilde{\boldsymbol{\varphi}}_{2}
$$

where $\tilde{\boldsymbol{\varphi}}_{/ \alpha}$ are the contravariant base vectors defined in this case as

$$
\begin{aligned}
& \tilde{\boldsymbol{\varphi}}_{\prime_{1}}=\lambda \boldsymbol{\varphi}_{\prime 2} \times \mathbf{t}_{3} \\
& \tilde{\boldsymbol{\varphi}}_{{ }^{\prime}}=-\lambda \boldsymbol{\varphi}_{\prime 1} \times \mathbf{t}_{3}
\end{aligned}
$$

Then one has

$$
\begin{aligned}
\delta \mathbf{t}_{3} & =\left(-\mathbf{t}_{3} \cdot \delta \boldsymbol{\varphi}_{\prime 1}\right) \tilde{\boldsymbol{\varphi}}_{\prime 1}+\left(-\mathbf{t}_{3} \cdot \delta \boldsymbol{\varphi}_{\prime 2}\right) \tilde{\boldsymbol{\varphi}}_{\prime 2} \\
& =-\sum_{J=1}^{3}\left[N_{\prime_{1}}^{J} \tilde{\boldsymbol{\varphi}}_{\prime_{1}}+N_{\prime 2}^{J} \tilde{\boldsymbol{\varphi}}_{\prime 2}\right]\left(\mathbf{t}_{3} \cdot \delta \mathbf{u}^{J}\right)
\end{aligned}
$$

Substituting this last expression into $\varphi_{r_{\alpha}}^{I} \cdot \delta \mathbf{t}_{3}$ one has
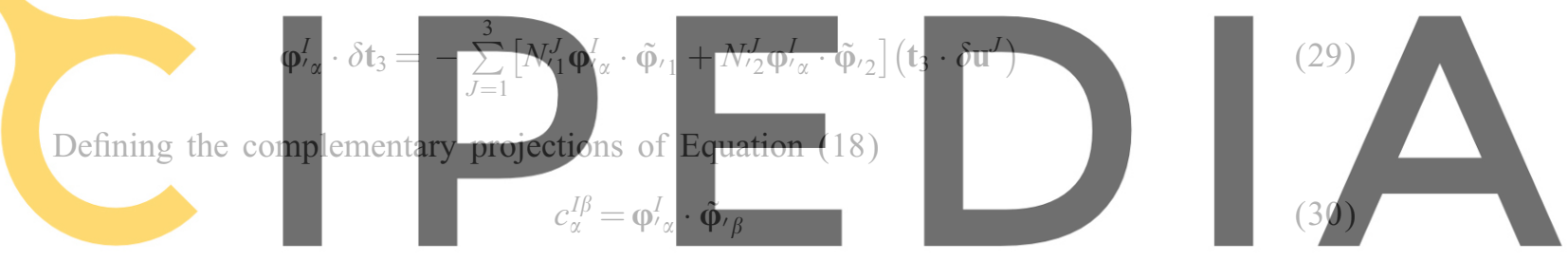

so that

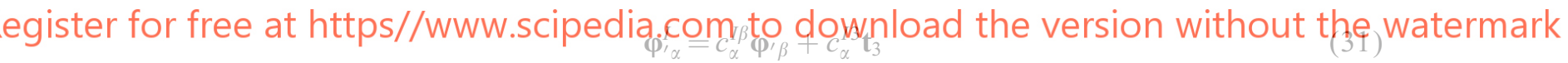

Replacing in the previous expression

$$
\boldsymbol{\varphi}_{{ }_{\alpha}}^{I} \cdot \delta \mathbf{t}_{3}=-\sum_{J=1}^{3}\left[N_{\prime_{1}}^{J} c_{\alpha}^{I 1}+N_{{ }_{2}}^{J} c_{\alpha}^{I 2}\right]\left(\mathbf{t}_{3} \cdot \delta \mathbf{u}^{J}\right)
$$

and finally, substituting these expressions into the variation of $\left(\mathbf{t}_{3} \cdot \boldsymbol{\varphi}_{\prime_{\alpha}}^{I}\right)$ one has

$$
\delta\left(\mathbf{t}_{3} \cdot \boldsymbol{\varphi}_{\prime \alpha}^{I}\right)=\sum_{J=1}^{3} N_{\prime_{\alpha}}^{J(I)} \mathbf{t}_{3} \cdot\left(\delta \mathbf{u}^{J}\right)^{I}-\sum_{J=1}^{3}\left[N_{\iota_{1}}^{J} c_{\alpha}^{I 1}+N_{\prime_{2}}^{J} c_{\alpha}^{12}\right]\left(\mathbf{t}_{3} \cdot \delta \mathbf{u}^{J}\right)
$$

The first term contributes to adjacent elements while the second term contributes to the main triangle only

$$
\delta\left[\begin{array}{l}
\chi_{11} \\
\chi_{22} \\
\chi_{12}
\end{array}\right]=\sum_{I=1}^{3} \sum_{J=1}^{3}\left[\begin{array}{cc}
N_{\prime 1}^{I} & 0 \\
0 & N_{\prime 2}^{I} \\
N_{\prime 2}^{I} & N_{\prime 1}^{I}
\end{array}\right]\left[\begin{array}{l}
N_{1}^{J(I)} \mathbf{t}_{3} \cdot\left(\delta \mathbf{u}^{J}\right)^{I}-\left[N_{\prime 1}^{J} c_{1}^{I 1}+N_{\prime_{2}}^{J} c_{1}^{I 2}\right]\left(\mathbf{t}_{3} \cdot \delta \mathbf{u}^{J}\right) \\
N_{\prime 2}^{J(I)} \mathbf{t}_{3} \cdot\left(\delta \mathbf{u}^{J}\right)^{I}-\left[N_{\prime_{1}}^{J} c_{2}^{I 1}+N_{\prime_{2}}^{J} c_{2}^{I 2}\right]\left(\mathbf{t}_{3} \cdot \delta \mathbf{u}^{J}\right)
\end{array}\right]
$$




\section{BOUNDARY CONDITIONS}

A special consideration must be done at the boundaries where there is no adjacent element to a side. The type of essential boundary condition referred to the normal rotation is crucial. The simplest choice is to neglect the contribution to the side rotations from the adjacent element missing in the patch in the evaluation of the curvatures via Equation (13) [14]. More precise changes can be, however, introduced to account for the different types of boundary conditions. For instance, when a fixed support (clamped boundary) or a symmetry plane is present, it is assumed that the contribution to the evaluation of curvatures of the clamped side (for example side $I$ ) has the following components (see expression (17)):

$$
\left[\begin{array}{l}
k_{11} \\
k_{22} \\
k_{12}
\end{array}\right]^{I}=-2\left[\begin{array}{cc}
N_{11}^{I} & 0 \\
0 & N_{12}^{I} \\
N_{12}^{I} & N_{11}^{I}
\end{array}\right]\left[\begin{array}{l}
n_{1}^{I} \\
n_{2}^{I}
\end{array}\right]\left(\mathbb{t}_{3} \cdot \varphi_{\prime n}^{(0)}\right)
$$

with $\varphi_{/ n}^{(0)}$, as the normal direction to the plane of symmetry (or plane of fixed support) referred to a local-to-the-boundary system (side $I$ ). In the reference configuration this contribution is zero, and as the shell deforms it will correctly take into account the rotation of the triangle.

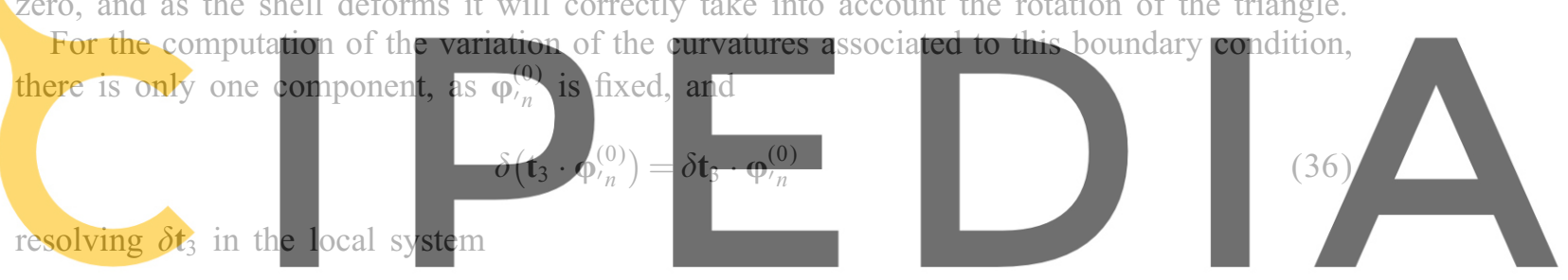

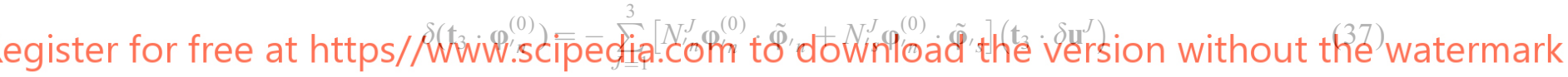

where $N_{i n}^{J}$ and $N_{i s}^{J}$ are shape function derivatives referred to the local boundary system. Note that $\boldsymbol{\varphi}_{{ }_{n}}^{(0)} \cdot \tilde{\boldsymbol{\varphi}}_{s}=0$; then, it follows that

$$
\delta\left(\mathbf{t}_{3} \cdot \boldsymbol{\varphi}_{\prime n}^{(0)}\right)=\boldsymbol{\varphi}_{\prime n}^{(0)} \cdot \delta t_{3 n} \tilde{\boldsymbol{\varphi}}_{\prime n}=-\boldsymbol{\varphi}_{\prime n}^{(0)} \cdot \tilde{\boldsymbol{\varphi}}_{\prime_{n}} \sum_{J=1}^{3} N_{\prime_{n}}^{J}\left(\mathbf{t}_{3} \cdot \delta \mathbf{u}^{J}\right)
$$

The final contribution of the clamped side $(I)$ to the curvature variation is

$$
\left[\begin{array}{l}
\delta \chi_{11} \\
\delta \chi_{22} \\
\delta \chi_{12}
\end{array}\right]^{I}=2\left[\begin{array}{cc}
N_{\prime 1}^{I} & 0 \\
0 & N_{\prime 2}^{I} \\
N_{\prime 2}^{I} & N_{\prime 1}^{I}
\end{array}\right]\left[\begin{array}{l}
n_{1}^{I} \\
n_{2}^{I}
\end{array}\right]\left[\begin{array}{ll}
n_{1}^{I} & n_{2}^{I}
\end{array}\right] \boldsymbol{\varphi}_{\prime n}^{(0)} \cdot \tilde{\boldsymbol{\varphi}}_{\prime_{n}} \sum_{J=1}^{3}\left[\begin{array}{c}
N_{\prime 1}^{J} \\
N_{\prime 2}^{J}
\end{array}\right]\left(\mathbf{t}_{3} \cdot \delta \mathbf{u}^{J}\right)
$$

For a simple supported (hinged) side, the problem is not completely defined. One may assume that the curvature normal to the side is zero, and consider a contribution of the missing side to introduce this constraint. As the change of curvature parallel to the side is zero along the hinged side, both things lead to zero curvatures in both directions. Denoting 
the contribution to curvatures of the existing sides $(J$ and $K)$ by

$$
\left[\begin{array}{l}
k_{11} \\
k_{22} \\
k_{12}
\end{array}\right]^{J-K}
$$

it can be easily shown that to set the normal curvature to zero the contribution of the simple supported side $(I)$ should be

$$
\left[\begin{array}{l}
k_{11} \\
k_{22} \\
k_{12}
\end{array}\right]^{I}=-\left[\begin{array}{ccc}
\left(n_{1}^{I}\right)^{4} & \left(n_{1}^{I}\right)^{2}\left(n_{2}^{I}\right)^{2} & \left(n_{1}^{I}\right)^{3} n_{2}^{I} \\
\left(n_{1}^{I}\right)^{2}\left(n_{2}^{I}\right)^{2} & \left(n_{2}^{I}\right)^{4} & n_{1}^{I}\left(n_{2}^{I}\right)^{3} \\
2\left(n_{1}^{I}\right)^{3} n_{2}^{I} & 2 n_{1}^{I}\left(n_{2}^{I}\right)^{3} & 2\left(n_{1}^{I}\right)^{2}\left(n_{2}^{I}\right)^{2}
\end{array}\right]^{2}\left[\begin{array}{l}
k_{11} \\
k_{22} \\
k_{12}
\end{array}\right]^{J-K}
$$

For the case of a triangle with two sides associated to hinged sides, the normal curvatures to both sides must be zero. Denoting by $\mathbf{n}^{I}$ and $\mathbf{n}^{J}$ the normal to the sides, and by $\mathbf{m}^{I}$ and $\mathbf{m}^{J}$ the dual base (associated to base $\mathbf{n}^{I}-\mathbf{n}^{J}$ ), the contribution from the hinged sides ( $I$ and $J)$ can be written as a function of the contribution of the only existing side $(K)$ :

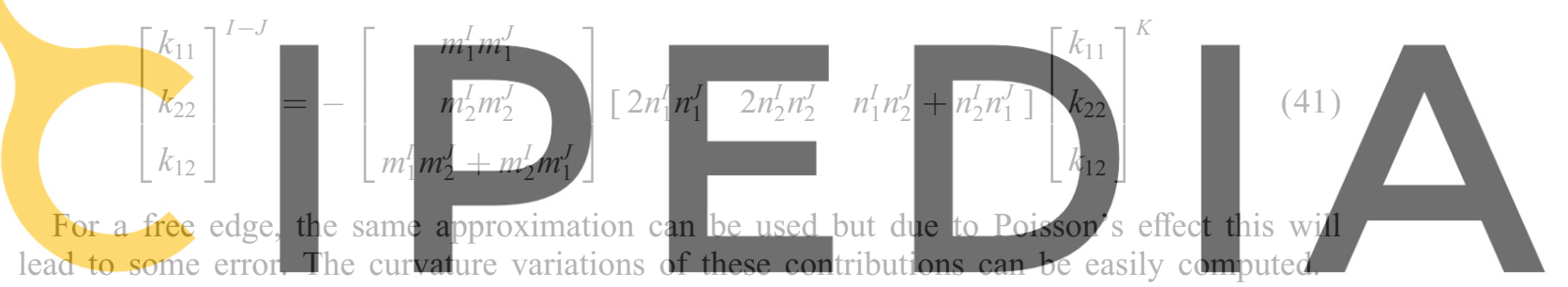

The stresses are computed using a Total Lagrangian Formulation. For a step $n$, the configuration $\varphi^{n}$ and the plastic strains $\varepsilon_{P}^{n}$ are known. The configuration $\varphi^{n}$ is obtained by adding the total displacements to the original configuration $\varphi^{n}=\varphi^{0}+\mathbf{u}^{n}$. The stresses are computed at each triangle using a single sampling (integration) point at the center and dividing the thickness into $N_{\mathrm{L}}$ layers. The plane stress state condition of the classical thin shell theory is assumed, so that for every layer three stress components are computed, $\left(\sigma_{11}, \sigma_{22}\right.$, and $\left.\sigma_{12}\right)$ referred to the local Cartesian system.

The computation of incremental stresses is as follows:

1. Evaluate incremental displacements: $\Delta \mathbf{u}^{n+1}$

2. Generate actual configuration $\boldsymbol{\varphi}^{n+1}=\boldsymbol{\varphi}^{n}+\Delta \mathbf{u}^{n+1}$

3. Compute metric tensor $a_{\alpha \beta}^{n+1}$ and curvatures $k_{\alpha \beta}^{n+1}$ for configuration $n+1$

4. Compute total and elastic deformations at each layer $k$

$$
\begin{aligned}
{[\boldsymbol{\varepsilon}]_{k}^{n+1} } & =\boldsymbol{\varepsilon}^{n+1}+z_{k} \chi^{n+1} \\
{\left[\boldsymbol{\varepsilon}_{e}\right]_{k}^{n+1} } & =[\boldsymbol{\varepsilon}]_{k}^{n+1}-\left[\boldsymbol{\varepsilon}_{p}\right]_{k}^{n}
\end{aligned}
$$


5. Compute trial elastic stresses at each layer $k$

$$
\sigma_{k}^{n+1}=\mathbf{C}\left[\boldsymbol{\varepsilon}_{e}\right]_{k}^{n+1}
$$

6. Check plasticity condition and return to plasticity surface if necessary, correct plastic strains $\left[\boldsymbol{\varepsilon}_{\mathrm{P}}\right]_{k}^{n+1}$ at each layer (small strain plasticity)

7. Compute generalized stresses

$$
\begin{aligned}
\mathbf{N}^{n+1} & =\frac{h^{(0)}}{N_{\mathrm{L}}} \sum_{k=1}^{N_{\mathrm{L}}} \sigma_{k}^{n+1} \\
\mathbf{M}^{n+1} & =\frac{h^{(0)}}{N_{\mathrm{L}}} \sum_{k=1}^{N_{\mathrm{L}}} \sigma_{k}^{n+1} z_{k}
\end{aligned}
$$

Note that $z_{k}$ is the current distance of the layer to the mid-surface and not the original distance. However, for small strain plasticity this distinction is not important.

This computation of stresses is adequate for an implicit scheme independent of the step size and it is exact for an elastic problem.

The residual forces are computed from the expression

$$
\delta \mathbf{u}^{\mathrm{T}} \mathbf{r}(\mathbf{u})=\int_{V_{0}} \delta \boldsymbol{\varepsilon}^{\mathrm{T}} \boldsymbol{\sigma} \mathrm{d} V_{0}
$$

For a shell theory, the above expression simplifies to

$$
\delta \mathbf{u}^{\mathrm{T}} \mathbf{r}(\mathbf{u})=\int_{A_{0}}\left(\delta \boldsymbol{\varepsilon}_{o}^{\mathrm{T}} \mathbf{N}+\delta \chi^{\mathrm{T}} \mathbf{M}\right) \mathrm{d} A_{0}
$$

and

$$
\mathbf{r}^{n+1}=A^{(0)}\left[\begin{array}{ll}
\mathbf{B}_{m}^{\mathrm{T}} & \mathbf{B}_{b}^{\mathrm{T}}
\end{array}\right]^{n+1}\left[\begin{array}{l}
\mathbf{N} \\
\mathbf{M}
\end{array}\right]^{n+1}
$$

\section{TANGENT STIFFNESS MATRIX}

For an implicit scheme used in conjunction with a Newton-type method the tangent stiffness matrix $\mathbf{K}$ is obtained as

$$
\delta \mathbf{u}^{\mathrm{T}} \mathbf{K} \Delta \mathbf{u}=\int_{A} \frac{\partial}{\partial \mathbf{u}}\left(\delta \boldsymbol{\varepsilon}^{\mathrm{T}} \mathbf{N}+\delta \chi^{\mathrm{T}} \mathbf{M}\right) \Delta \mathbf{u} \mathrm{d} A
$$

The two following contributions are identified in Equation (44).

\subsection{Material part}

$$
\begin{aligned}
\delta \mathbf{u}^{\mathrm{T}} \mathbf{K}^{\mathrm{M}} \Delta \mathbf{u} & =\int_{A}\left(\delta \boldsymbol{\varepsilon}^{\mathrm{T}} \frac{\partial}{\partial \mathbf{u}} \mathbf{N}+\delta \boldsymbol{\chi}^{\mathrm{T}} \frac{\partial}{\partial \mathbf{u}} \mathbf{M}\right) \Delta \mathbf{u} \mathrm{d} A \\
& =\delta \mathbf{u}^{\mathrm{T}} A^{(0)} \mathbf{B}^{\mathrm{T}} \mathbf{D}_{\mathrm{EP}} \mathbf{B} \Delta \mathbf{u}
\end{aligned}
$$


where $\mathbf{D}_{\mathrm{EP}}$ is the elastoplastic constitutive matrix obtained by integrating across the thickness the algorithmic tangent matrix $\mathbf{C}_{\mathrm{EP}}$. This matrix relates strain increments with stress increments in the plane stress state of each layer.

$$
\mathbf{D}_{\mathrm{EP}}=\frac{h^{(0)}}{N_{\mathrm{L}}} \sum_{k=1}^{N_{\mathrm{L}}}\left[\begin{array}{cc}
\mathbf{C}_{\mathrm{EP}}^{k} & z_{k} \mathbf{C}_{\mathrm{EP}}^{k} \\
z_{k} \mathbf{C}_{\mathrm{EP}}^{k} & z_{k}^{2} \mathbf{C}_{\mathrm{EP}}^{k}
\end{array}\right]
$$

\subsection{Geometric part}

$$
\delta \mathbf{u}^{\mathrm{T}} \mathbf{K}^{\mathrm{G}} \Delta \mathbf{u}=\int_{A}\left(\frac{\partial}{\partial \mathbf{u}}\left(\delta \boldsymbol{\varepsilon}^{\mathrm{T}}\right) \mathbf{N}+\frac{\partial}{\partial \mathbf{u}}\left(\delta \chi^{\mathrm{T}}\right) \mathbf{M}\right) \Delta \mathbf{u} \mathrm{d} A
$$

Membrane and bending contributions are considered separately in the computation of $\mathbf{K}^{\mathrm{G}}$. The membrane contribution is standard

$$
\begin{aligned}
\delta \mathbf{u}^{\mathrm{T}} \mathbf{K}_{m}^{\mathrm{G}} \Delta \mathbf{u}= & \int_{A} \frac{\partial}{\partial \mathbf{u}}\left(\delta \boldsymbol{\varepsilon}^{\mathrm{T}}\right) \mathbf{N} \Delta \mathbf{u} \mathrm{d} A \\
= & A^{(0)} \sum_{I=1}^{3} \sum_{J=1}^{3}\left[N_{\prime_{1}}^{I} N_{\prime_{1}}^{J} N_{11}+N_{\prime_{2}}^{I} N_{\prime_{2}}^{J} N_{22}\right. \\
& \left.+\left(N_{\prime_{1}}^{I} N_{\prime_{2}}^{J}+N_{\prime_{2}}^{I} N_{\prime_{1}}^{J}\right) N_{12}\right] \delta \mathbf{u}^{J} \cdot \Delta \mathbf{u}^{I} \\
= & A^{(0)} \sum_{I=1}^{3} \sum_{J=1}^{3} \delta \mathbf{u}^{I}\left[\begin{array}{ll}
N_{\prime_{1}}^{J} & N_{\prime_{2}}^{J}
\end{array}\right]\left[\begin{array}{ll}
N_{11} & N_{12} \\
N_{21} & N_{22}
\end{array}\right]\left[\begin{array}{l}
N_{\prime_{1}}^{J} \\
N_{{ }^{\prime}}^{J}
\end{array}\right] \Delta \mathbf{u}^{J}
\end{aligned}
$$

For the bending part more algebra is involved. Recalling Equations (16) and (17), the contribution to the geometric stiffness due to bending can be written as

$$
\begin{aligned}
\delta \mathbf{u}^{\mathrm{T}} \mathbf{K}_{\mathrm{b}}^{\mathrm{G}} \Delta \mathbf{u} & =A^{(0)}\left[\begin{array}{lll}
M_{11} & M_{22} & M_{12}
\end{array} \sum_{I=1}^{3}\left[\begin{array}{cc}
N_{\prime 1}^{I} & 0 \\
0 & N_{\prime 2}^{I} \\
N_{\prime 2}^{I} & N_{\prime 1}^{I}
\end{array}\right]\left[\begin{array}{l}
\Delta\left[\delta\left(\mathbf{t}_{3} \cdot \boldsymbol{\varphi}_{\prime 1}^{I}\right)\right] \\
\Delta\left[\delta\left(\mathbf{t}_{3} \cdot \boldsymbol{\varphi}_{\prime 2}^{I}\right)\right]
\end{array}\right]\right. \\
& =A^{(0)} \sum_{I=1}^{3}\left[\begin{array}{ll}
N_{\prime 1}^{I} & N_{\prime 2}^{I}
\end{array}\right]\left[\begin{array}{ll}
M_{11} & M_{12} \\
M_{12} & M_{22}
\end{array}\right]\left[\begin{array}{l}
\Delta\left[\delta\left(\mathbf{t}_{3} \cdot \boldsymbol{\varphi}_{\prime 1}^{I}\right)\right] \\
\Delta\left[\delta\left(\mathbf{t}_{3} \cdot \boldsymbol{\varphi}_{\prime 2}^{I}\right)\right]
\end{array}\right]
\end{aligned}
$$

Or, alternatively

$$
\delta \mathbf{u}^{\mathrm{T}} \mathbf{K}_{\mathrm{b}}^{\mathrm{G}} \Delta \mathbf{u}=A^{(0)} \sum_{I=1}^{3}\left[\begin{array}{ll}
N_{\prime 1}^{I} & N_{\prime 2}^{I}
\end{array}\right]\left[\begin{array}{ll}
M_{11} & M_{12} \\
M_{12} & M_{22}
\end{array}\right]\left[\begin{array}{l}
n_{1}^{I} \\
n_{2}^{I}
\end{array}\right] \Delta\left[\delta\left(\mathbf{t}_{3} \cdot \boldsymbol{\varphi}_{\prime n}^{I}\right)\right]
$$


Then one has to compute

$$
\begin{aligned}
\Delta\left[\delta\left(\mathbf{t}_{3} \cdot \boldsymbol{\varphi}_{\prime \alpha}^{I}\right)\right] & =\Delta\left[\delta \mathbf{t}_{3} \cdot \boldsymbol{\varphi}_{\prime \alpha}^{I}+\mathbf{t}_{3} \cdot \delta \boldsymbol{\varphi}_{\prime \alpha}^{I}\right] \\
& =\Delta\left(\delta \mathbf{t}_{3}\right) \cdot \boldsymbol{\varphi}_{{ }^{\prime} \alpha}^{I}+\Delta \mathbf{t}_{3} \cdot \delta \boldsymbol{\varphi}_{\prime \alpha}^{I}+\delta \mathbf{t}_{3} \cdot \Delta \boldsymbol{\varphi}_{{ }^{\prime} \alpha}^{I}+\mathbf{t}_{3} \cdot \Delta\left(\delta \boldsymbol{\varphi}^{I}{ }^{I}\right)
\end{aligned}
$$

Note that the last term is zero $\left(\Delta\left(\delta \varphi_{\prime \alpha}^{I}\right)=0\right)$. The second and third terms are of the form (they lead to corresponding symmetric components)

$$
\begin{aligned}
\delta \mathbf{t}_{3} \cdot \Delta \boldsymbol{\varphi}_{\prime \alpha}^{I} & =\left\{-\sum_{J=1}^{3}\left[N_{\prime_{1}}^{J} \tilde{\boldsymbol{\varphi}}_{\prime_{1}}+N_{\prime_{2}}^{J} \tilde{\boldsymbol{\varphi}}, 2_{2}\right]\left(\mathbf{t}_{3} \cdot \delta \mathbf{u}^{J}\right)\right\} \cdot\left\{\sum_{K=1}^{3} N_{\prime_{\alpha}}^{K(I)} \Delta \mathbf{u}^{K(I)}\right\} \\
& =-\sum_{J=1}^{3} \sum_{K=1}^{3}\left(\delta \mathbf{u}^{J}\right)^{\mathrm{T}}\left[N_{\prime_{1}}^{J}\left(\mathbf{t}_{3} \otimes \tilde{\boldsymbol{\varphi}}_{{ }_{1}}\right)+N_{\prime_{2}}^{J}\left(\mathbf{t}_{3} \otimes \tilde{\boldsymbol{\varphi}}_{\prime 2}\right)\right] N_{\prime_{\alpha}}^{K(I)} \Delta \mathbf{u}^{K(I)}
\end{aligned}
$$

This term relates the nodes in the main triangular element with the nodes in adjacent elements. The contribution of the second and third terms of Equation (51) leads to the following expression for the bending part of the tangent stiffness matrix:

$$
\begin{aligned}
\delta \mathbf{u}^{\mathrm{T}} \mathbf{K}_{\mathrm{b} 1}^{\mathrm{G}} \Delta \mathbf{u}= & A^{(0)}\left(-\sum_{J=1}^{3}\left(\delta \mathbf{u}^{J}\right)^{\mathrm{T}}\left[\begin{array}{ll}
N_{\prime 1}^{J} & N_{\prime 2}^{J}
\end{array}\right]\left[\begin{array}{l}
\mathbf{t}_{3} \otimes \tilde{\boldsymbol{\varphi}}_{\prime 1} \\
\mathbf{t}_{3} \otimes \tilde{\boldsymbol{\varphi}}_{\prime 2}
\end{array}\right]\right) \\
& \sum_{I=1}^{3}\left[\begin{array}{ll}
N_{\prime 1}^{I} & N_{\prime 2}^{I}
\end{array}\right]\left[\begin{array}{ll}
M_{11} & M_{12} \\
M_{12} & M_{22}
\end{array}\right] \sum_{K=1}^{3}\left[\begin{array}{l}
N_{\prime 1}^{K(I)} \\
N_{\prime 2}^{K(I)}
\end{array}\right] \Delta \mathbf{u}^{K(I)}
\end{aligned}
$$

The first term on the r.h.s. of Equation (51) $\Delta\left(\delta \mathbf{t}_{3}\right) \cdot \boldsymbol{\varphi}_{\prime n}^{I}$ (the form of Equation (50) is more convenient here) is rather complex. In the appendix it is shown that $\Delta\left(\delta \mathbf{t}_{3}\right)$ can be resolved in the convective base, leading to

$$
\begin{aligned}
& \Delta\left(\delta \mathbf{t}_{3}\right)=\left[\Delta\left(\delta \mathbf{t}_{3}\right)\right]_{1} \tilde{\boldsymbol{\varphi}}_{{ }_{1}}+\left[\Delta\left(\delta \mathbf{t}_{3}\right)\right]_{2} \tilde{\boldsymbol{\varphi}}_{{ }^{\prime}}+\left[\Delta\left(\delta \mathbf{t}_{3}\right)\right]_{3} \mathbf{t}_{3} \\
& =-\left(\tilde{\boldsymbol{\varphi}}_{\alpha \alpha} \cdot \tilde{\boldsymbol{\varphi}}_{\beta \beta}\right)\left[\delta \boldsymbol{\varphi}^{\mathrm{T} \alpha}\left(\mathbf{t}_{3} \otimes \mathbf{t}_{3}\right) \Delta \boldsymbol{\varphi}^{\prime} \beta\right] \mathbf{t}_{3} \\
& +\left[\delta \boldsymbol{\varphi}_{\prime_{\alpha}}^{\mathrm{T}}\left(\mathbf{t}_{3} \otimes \tilde{\boldsymbol{\varphi}}_{\alpha \alpha}\right) \Delta \boldsymbol{\varphi}_{\beta}\right] \tilde{\boldsymbol{\varphi}}_{\beta}+\left[\delta \boldsymbol{\varphi}^{\mathrm{T}}\left(\tilde{\boldsymbol{\varphi}}_{\beta} \otimes \mathbf{t}_{3}\right) \Delta \boldsymbol{\varphi}_{\beta}\right] \tilde{\boldsymbol{\varphi}}_{\alpha}
\end{aligned}
$$

Vector $\Delta\left(\delta \mathbf{t}_{3}\right)$ will be used in conjunction with $\boldsymbol{\varphi}_{\prime n}^{I}$, then

$$
\begin{aligned}
& \Delta\left(\delta \mathbf{t}_{3}\right) \cdot \boldsymbol{\varphi}_{\prime n}^{I}=-\left(\tilde{\boldsymbol{\varphi}}_{{ }^{\prime} \alpha} \cdot \tilde{\boldsymbol{\varphi}}_{\beta}\right)\left[\delta \boldsymbol{\varphi}^{\mathrm{T} \alpha}\left(\mathbf{t}_{3} \otimes \mathbf{t}_{3}\right) \Delta \boldsymbol{\varphi}^{\prime} \beta\right] \mathbf{t}_{3} \cdot \boldsymbol{\varphi}_{\prime^{\prime}}^{I} \\
& +\left[\delta \boldsymbol{\varphi}^{\mathrm{T}}\left(\mathbf{t}_{3} \otimes \tilde{\boldsymbol{\varphi}}_{{ }^{\prime}}\right) \Delta \boldsymbol{\varphi}^{\prime} \beta\right] \tilde{\boldsymbol{\varphi}}_{\beta} \cdot \boldsymbol{\varphi}_{\prime_{n}}^{I} \\
& +\left[\delta \boldsymbol{\varphi}_{\prime_{\alpha}}^{\mathrm{T}}\left(\tilde{\boldsymbol{\varphi}}_{{ }^{\prime} \beta} \otimes \mathbf{t}_{3}\right) \Delta \boldsymbol{\varphi}^{\prime} \beta\right] \tilde{\boldsymbol{\varphi}}_{{ }_{\alpha}} \cdot \boldsymbol{\varphi}_{\prime_{n}}^{I}
\end{aligned}
$$




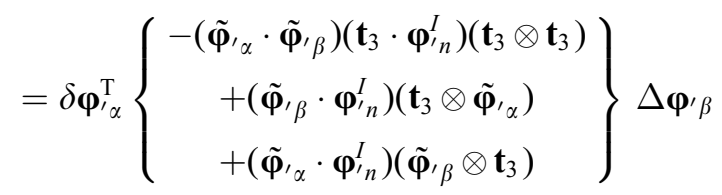

$$
\begin{aligned}
& \Delta\left(\delta \mathbf{t}_{3}\right) \cdot \boldsymbol{\varphi}_{\prime n}^{I}=\sum_{J=1}^{3} \sum_{K=1}^{3} \sum_{\alpha=1}^{2} \sum_{\beta=1}^{2} N_{\alpha \alpha}^{J} N_{\prime \beta}^{K}\left[\delta \mathbf{u}^{J}\right]^{\mathrm{T}} \mathbf{P}_{a \beta}^{I} \Delta \mathbf{u}^{K}
\end{aligned}
$$

with

$$
\mathbf{P}_{\alpha \beta}^{I}=\left[-a^{\alpha \beta} c^{I 3}\left(\mathbf{t}_{3} \otimes \mathbf{t}_{3}\right)+c^{I \beta}\left(\mathbf{t}_{3} \otimes \tilde{\boldsymbol{\varphi}}_{\alpha_{\alpha}}\right)+c^{I \alpha}\left(\tilde{\boldsymbol{\varphi}}_{\prime_{\beta}} \otimes \mathbf{t}_{3}\right)\right]
$$

where the definition of the contravariant metric tensor has been used, i.e.

$$
a^{\alpha \beta}=\tilde{\boldsymbol{\varphi}}_{\alpha_{\alpha}} \cdot \tilde{\boldsymbol{\varphi}}_{\beta}=\left(a_{\alpha \beta}\right)^{-1}
$$

Finally,

$$
\begin{aligned}
\delta \mathbf{u}^{\mathrm{T}} \mathbf{K}_{\mathrm{b} 2}^{\mathrm{G}} \Delta \mathbf{u} & =A^{(0)} \sum_{I=1}^{3}\left[\begin{array}{ll}
N_{\prime 1}^{I} & \left.N_{\prime 2}^{I}\right]
\end{array}\right]\left[\begin{array}{ll}
M_{11} & M_{12} \\
M_{12} & M_{22}
\end{array}\right]\left[\begin{array}{l}
n_{1}^{I} \\
n_{2}^{I}
\end{array}\right]\left[\Delta\left(\delta \mathbf{t}_{3}\right) \cdot \boldsymbol{\varphi}_{{ }_{n}}^{I}\right] \\
& =A^{(0)} \sum_{I=1}^{3} \sum_{\gamma=1}^{2} \sum_{\delta=1}^{2}\left(M_{\gamma \delta} N_{\prime \delta}^{I} n_{\gamma}^{I}\right) \sum_{J=1}^{3} \sum_{K=1}^{3} \sum_{\alpha=1}^{2} \sum_{\beta=1}^{2} N_{I_{\alpha}}^{J} N_{\prime \beta}^{K}\left[\delta \mathbf{u}^{J}\right]^{\mathrm{T}} \mathbf{P}_{a \beta}^{I} \Delta \mathbf{u}^{K}
\end{aligned}
$$

Equation (59) has components only in nodes of the main element.

Matrix $\mathbf{K}_{\mathrm{b}}^{\mathrm{G}}$ is computed by adding up the contributions from $\mathbf{K}_{\mathrm{b} 1}^{\mathrm{G}}$ and $\mathbf{K}_{\mathrm{b} 2}^{\mathrm{G}}$ deduced from Equations (53) and (59), respectively.

\section{LARGE STRAIN FORMULATION}

To consider large strain plasticity, an adequate definition of strain and stress measures must be considered. For this, first the position of any point is written as

$$
\mathbf{x}\left(\xi_{1}, \xi_{2}, \zeta\right)=\boldsymbol{\varphi}\left(\xi_{1}, \xi_{2}\right)+\zeta \lambda \mathbf{t}_{3}
$$

where $\xi_{1}, \xi_{2}$ are curvilinear local coordinates over the middle surface, and $\zeta$ is the original distance of the point to the middle surface. With this definition the product $\zeta \lambda$ is the actual distance of the point to the middle surface. 
The deformation gradient can be written as

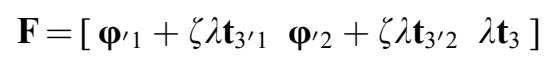

The product $\mathbf{F}^{\mathrm{T}} \mathbf{F}=\mathbf{U}^{2}$ (with $\mathbf{U}$ being the right stretch tensor) is

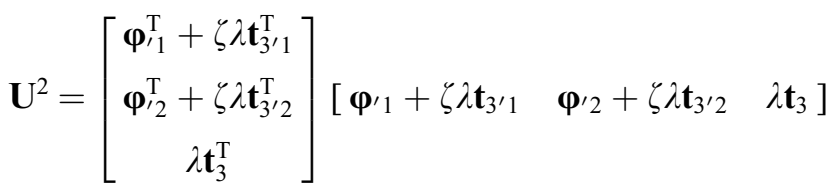

$$
\begin{aligned}
& =\left[\begin{array}{ccc}
\boldsymbol{\varphi}^{\prime} 1 \cdot \boldsymbol{\varphi}_{\prime 1} & \boldsymbol{\varphi}_{11} \cdot \boldsymbol{\varphi}_{22} & 0 \\
\boldsymbol{\varphi}^{\prime} 1 \cdot \boldsymbol{\varphi}_{22} & \boldsymbol{\varphi}_{22} \cdot \boldsymbol{\varphi}_{22} & 0 \\
0 & 0 & \lambda^{2}
\end{array}\right] \\
& +\zeta \lambda\left[\begin{array}{ccc}
2 \boldsymbol{\varphi}^{\prime} 1 \cdot \mathbf{t}_{3^{\prime} 1} & \boldsymbol{\varphi}^{\prime 1} \cdot \mathbf{t}_{3^{\prime} 2}+\boldsymbol{\varphi}^{\prime 2} \cdot \mathbf{t}_{3^{\prime} 1} & 0 \\
\boldsymbol{\varphi}^{\prime} 1 \cdot \mathbf{t}_{3^{\prime} 2}+\boldsymbol{\varphi}^{\prime 2} \cdot \mathbf{t}_{3^{\prime} 1} & \boldsymbol{\varphi}^{\prime 2} \cdot \mathbf{t}_{3^{\prime} 2} & 0 \\
0 & 0 & 0
\end{array}\right] \\
& +\zeta^{2} \lambda^{2}\left[\begin{array}{ccc}
\mathbf{t}_{3^{\prime} 1} \cdot \mathbf{t}_{3^{\prime} 1} & \mathbf{t}_{3^{\prime} 1} \cdot \mathbf{t}_{3^{\prime} 2} & 0 \\
\mathbf{t}_{3^{\prime} 1} \cdot \mathbf{t}_{3^{\prime 2}} & \mathbf{t}_{3^{\prime 2} 2} \cdot \mathbf{t}_{3^{\prime} 2} & 0 \\
0 & 0 & 0
\end{array}\right]
\end{aligned}
$$

Neglecting the term associated to $\zeta^{2}$ and introducing the definition of the metric tensor $a_{\alpha \beta}$ at the middle surface and of the curvatures $k_{\alpha \beta}$ gives

$$
\mathbf{U}^{2}=\left[\begin{array}{ccc}
a_{11}+2 k_{11} \zeta \lambda & a_{12}+2 k_{12} \zeta \lambda & 0 \\
a_{12}+2 k_{12} \zeta \lambda & a_{22}+2 k_{22} \zeta \lambda & 0 \\
0 & 0 & \lambda^{2}
\end{array}\right]
$$

For computational convenience, the following approximate expression (which is exact for initially flat surfaces) will be adopted

$$
\mathbf{U}^{2}=\left[\begin{array}{ccc}
a_{11}+2 \chi_{11} \zeta \lambda & a_{12}+2 \chi_{12} \zeta \lambda & 0 \\
a_{12}+2 \chi_{12} \zeta \lambda & a_{22}+2 \chi_{22} \zeta \lambda & 0 \\
0 & 0 & \lambda^{2}
\end{array}\right]
$$

where $\chi_{i j}$ are the changes of curvatures of the middle surface.

The definition of an objective strain measure requires the computation of the eigenvalues $\left(\lambda_{\alpha}\right)$ of the right stretch tensor $\mathbf{U}$ and the associated eigenvectors $\mathbf{r}_{\alpha}$. It is clear that $\mathbf{U}$ and $\mathbf{U}^{2}$ have the same eigenvectors and that the eigenvalues of $\mathbf{U}$ can be obtained taking the square root of the eigenvalues of $\mathbf{U}^{2}$. An immediate eigenpair of $\mathbf{U}$ is $\lambda_{3}=\lambda$ and $\mathbf{r}_{3}=(0,0,1)$; the 
other two pairs define the principal strain directions in the plane. The Hencky (logarithmic) strains will be adopted

$$
\mathbf{E}=\left[\begin{array}{ccc}
\varepsilon_{11} & \varepsilon_{21} & 0 \\
\varepsilon_{12} & \varepsilon_{22} & 0 \\
0 & 0 & \varepsilon_{33}
\end{array}\right]=\sum_{\alpha=1}^{3} \ln \left(\lambda_{\alpha}\right) \mathbf{r}_{\alpha} \otimes \mathbf{r}_{\alpha}
$$

Assuming small elastic strains, an additive decomposition of the elastic and plastic strains can also be adopted

$$
\mathbf{E}=\mathbf{E}^{\mathrm{e}}+\mathbf{E}^{\mathrm{p}}
$$

Consistently, the Hencky stress tensor as stress measure will be used, and a linear (constant) isotropic "plane stress" relation between elastic strains and stresses will be assumed

$$
\begin{gathered}
\mathbf{T}=\mathbf{C E}^{\mathrm{e}} \\
\left\{\begin{array}{c}
T_{11} \\
T_{22} \\
T_{12}
\end{array}\right\}=\frac{E}{\left(1-v^{2}\right)}\left[\begin{array}{ccc}
1 & v & 0 \\
v & 1 & 0 \\
0 & 0 & \frac{1-v}{2}
\end{array}\right]\left[\begin{array}{c}
\varepsilon_{11}^{\mathrm{e}} \\
\varepsilon_{22}^{\mathrm{e}} \\
2 \varepsilon_{12}^{\mathrm{e}}
\end{array}\right]
\end{gathered}
$$

The constitutive equations are integrated using a standard radial return algorithm for the von Mises yield criteria with non-linear isotropic hardening.

Finally, one needs to obtain the second Piola-Kirchhoff stresses (S) for the evaluation of the residual forces in the total Lagrangian formulation employed. Defining the rotated tensors as

$$
\begin{aligned}
\mathbf{T}_{\mathrm{L}} & =\mathbf{R}_{\mathrm{L}}^{\mathrm{T}} \mathbf{T} \mathbf{R}_{\mathrm{L}} \\
\mathbf{S}_{\mathrm{L}} & =\mathbf{R}_{\mathrm{L}}^{\mathrm{T}} \mathbf{S R}_{\mathrm{L}}
\end{aligned}
$$

where $\mathbf{R}_{\mathrm{L}}$ is the rotation tensor obtained from the eigenvectors of $\mathbf{U}$

$$
\mathbf{R}_{\mathrm{L}}=\left[\begin{array}{lll}
\mathbf{r}_{1} & \mathbf{r}_{2} & \mathbf{r}_{3}
\end{array}\right]
$$

The relation between the second Piola-Kirchhoff stresses and Hencky stresses is (see for example Reference [18])

$$
\begin{aligned}
& {\left[S_{\mathrm{L}}\right]_{\alpha \alpha}=\frac{1}{\lambda_{\alpha}^{2}}\left[T_{\mathrm{L}}\right]_{\alpha \alpha}} \\
& {\left[S_{\mathrm{L}}\right]_{\alpha \beta}=\frac{\ln \left(\lambda_{\alpha} / \lambda_{\beta}\right)}{\frac{1}{2}\left(\lambda_{\alpha}^{2}-\lambda_{\beta}^{2}\right)}\left[T_{\mathrm{L}}\right]_{\alpha \beta}}
\end{aligned}
$$

The second Piola-Kirchhoff stress tensor can therefore be computed as

$$
\mathbf{S}=\mathbf{R}_{\mathrm{L}} \mathbf{S}_{\mathrm{L}} \mathbf{R}_{\mathrm{L}}^{\mathrm{T}}
$$

A simplification might be considered here assuming that $\mathbf{U}$ and $\mathbf{T}$ are colinear, that is, that they diagonalize in the same base. In this case, Equation (71) simplifies to

$$
\mathbf{S}=\mathbf{T U}^{-2}
$$



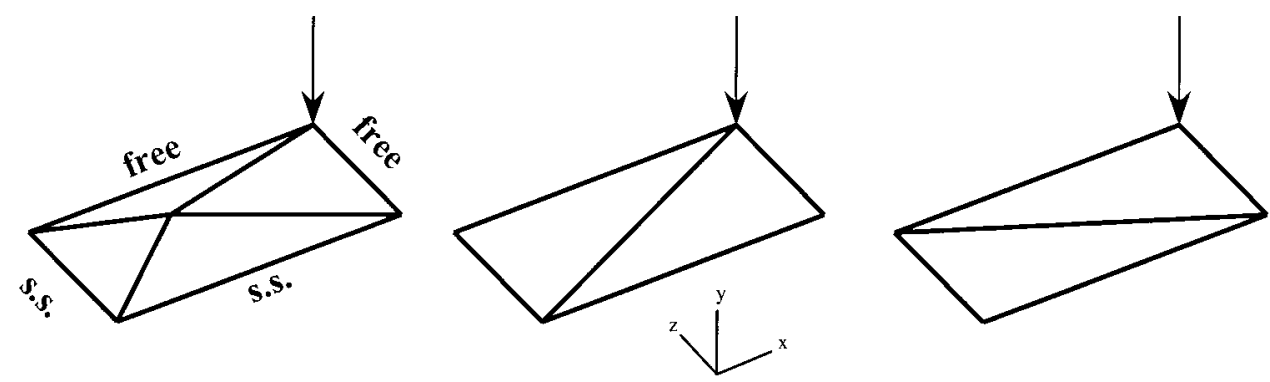

Figure 3. Patch test for a constant twisting moment. Exact results for displacements nd stresses are obtained.

This approximation is exact for elastic processes (isotropic materials) and perhaps for inelastic processes with radial loading, but not for general elastic-plastic processes.

The computation of the generalized stresses is performed integrating in the (original) thickness the second Piola-Kirchhoff stresses but using for the evaluation of moments the current distance to the middle surface as described earlier.

\section{EXAMPLES}

The accuracy and efficiency of the BST (for basic shell triangle) element will be tested in a number of linear and non-linear examples of plate and shell analysis.

\subsection{Linear examples}

9.1.1. Patch test. The membrane behaviour does not deserve any consideration as it corresponds to the well-known constant strain triangle. For the bending behaviour, as the formulation does not allow moments as loads (the element has translational DOFs only), it is not possible to test an isolated patch under loads leading to a constant bending moment. A constant twisting moment can be imposed through a point load on the corner of a rectangular plate with two (consecutive) sides simple supported and the other two sides free. Figure 3 shows three patch configurations that lead to the correct results both in displacements and stresses.

9.1.2. Square plate. A square plate under uniform load, with either hinged or clamped support is considered in this section. It is intended to assess the convergence properties of the BST element for structured and unstructured meshes. Due to symmetry one-quarter of the plate is modelled in all cases. Figure 4 shows the type of unstructured and structured meshes considered. For the case of structured meshes two orientations are possible.

Table I lists values of the normalized displacement of the plate mid-point for both boundary conditions for the three types of meshes considered. Convergence rates for the structured meshes are better than typical ones of linear triangles. Note that for plate bending analysis only one DOF per node is required [10, 14]. For example in Reference [19] for the simple supported case and a mesh with 208 DOFs, the two elements presented in that paper show an 


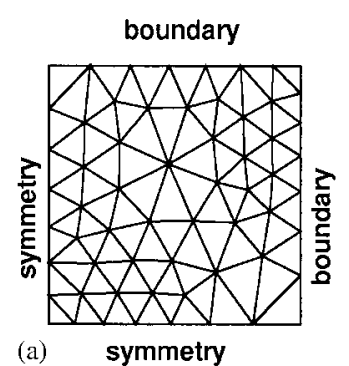

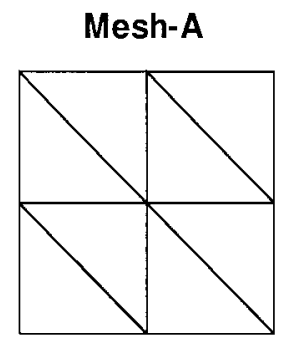

(b)
Mesh-B

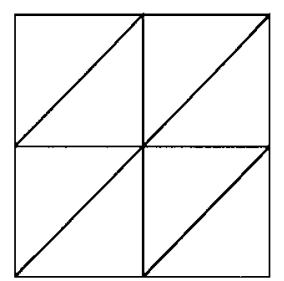

(c)

Figure 4. Square plate under uniform load, $E=10.92 \mathrm{E}^{5}, v=0.3, L / h=1000$ : (a) unstructured mesh;

(b) structured mesh, orientation A; and (c) structured mesh, orientation B.

Table I. Normalized results for the square plate under uniform load.

\begin{tabular}{|c|c|c|c|c|c|c|c|}
\hline \multirow[b]{2}{*}{ NDOF } & \multicolumn{2}{|c|}{ Mesh-A } & \multicolumn{2}{|c|}{ Mesh-B } & \multicolumn{3}{|c|}{ Unstructured } \\
\hline & s.s. & Clamped & s.s. & Clamped & NDOF & s.s. & Clamped \\
\hline 1 & 0.9496 & 2.0667 & 2.1364 & 5.9044 & & & \\
\hline 4 & 1.0050 & 1.5090 & 1.2315 & 2.0531 & 11 & 1.0034 & 1.2687 \\
\hline 16 & 1.0029 & 1.1573 & 1.0486 & 1.2418 & 46 & 0.9529 & 0.9969 \\
\hline 64 & 1.0005 & 1.0432 & 1.0112 & 1.0606 & 288 & 0.9807 & 0.9955 \\
\hline 256 & 1.0000 & 1.0138 & 1.0027 & 1.0179 & 762 & 0.9828 & 0.9778 \\
\hline 1024 & 1.0000 & 1.0066 & 1.0006 & 1.0076 & 3042 & 0.9873 & 0.9867 \\
\hline
\end{tabular}

Table II. Cylindrical roof under uniform dead load. Normalized displacements.

\begin{tabular}{rccccc}
\hline & \multicolumn{2}{c}{ Point-A } & & \multicolumn{2}{c}{ Point-B } \\
\cline { 2 - 3 } \cline { 6 - 6 } NDOF & Mesh-A & Mesh-B & & Mesh-A & Mesh-B \\
\hline 16 & 0.7416 & 0.4042 & & 1.3523 & 0.8978 \\
56 & 0.7401 & 0.6164 & & 0.7559 & 0.6824 \\
208 & 0.8849 & 0.8501 & & 0.8827 & 0.8637 \\
800 & 0.9652 & 0.9563 & & 0.9639 & 0.9586 \\
3136 & 0.9910 & 0.9888 & 0.9899 & 0.9885 \\
\hline
\end{tabular}

error in the central displacement between 1.5 per cent (mesh A) and 0.6 per cent (mesh B). For the present element with only 64 DOFs the errors are of 0.05 per cent (mesh A) and 1.1 per cent (mesh B). Unstructured meshes show a slower convergence rate. The accuracy can be improved here by using adaptive mesh refinement $[13,14]$.

9.1.3. Cylindrical roof. This cylindrical shell roof, loaded vertically by dead weight, is shown in Figure 5. It is supported by rigid diaphragms at the ends. Only one-quarter of the roof is modelled due to symmetry. Structured meshes with the two possible orientations (figure shows mesh-A orientation) were considered to assess convergence. Table II shows normalized vertical displacements at the crown (Point A) and at the midpoint of the free edge. The exact 
values $u_{\mathrm{A}}=0.5407$ and $u_{\mathrm{B}}=-3.610$ used for normalizing the present results were taken from Reference [20].

In this membrane dominated problem, the convergence is typical of the constant strain triangle. The advantage of the present formulation is that the element has only 3 DOFs per node. For example, a mesh with 16 elements per side, in the present formulation, has 800 DOFs while for elements combining the constant strain triangle for membrane and the Discrete Kirchhoff Triangle for bending (CST-DKT) [21] the number of DOFs is between 1328 (5 DOFs per node) and 1584 (6 DOFs per node), with similar convergence properties.

\subsection{Non-linear examples}

Six examples are included to assess the performance of the present formulation of the rotationfree BST element in the non-linear range. The first three examples consider elastic material with large displacements and rotations. Then plasticity with small strains is considered. The last two examples are well-known sheet metal forming benchmarks. Computations for the last two examples are performed with an explicit formulation due to severe frictional contact conditions. Contact is ensured by means of a standard penalty formulation and friction is considered via a linear Coulomb model.

9.2.1. Pear-shaped cylinder. The elastic large deflection response of a pear-shaped cylinder under end shortening, was proposed as a benchmark test in Reference [22]. The main features of the benchmark are elastic large displacements and rotations, initial softening behaviour followed by stiffening up to final buckling (collapse) load, stress redistribution and varying interaction of bending, membrane and shear effects. Figure 6(a) shows the geometrical definition of the cylinder cross-section and material data. Due to symmetry, a quarter of the geometry is discretized with 1920 BST elements involving 1067 nodes and 2794 DOFs. The uniform end-shortening is applied using a displacement control arc-length in 45 equal steps until collapse is well-developed (maximum end-shortening is 0.45 ). Figure 6(b) shows a plot of the total axial load as a function of the normal displacement at the point denoted as node 1 , located at the middle of the length of the cylinder, on the plane of symmetry, flat side. The results are compared with "target" results obtained with the program ABAQUS [23]. The value of the collapse load for the present formulation (2504) is in the range suggested ( $2437 \pm 3$ per cent).

9.2.2. L-shaped plate strip. The stability of this model problem (see Figure 7(a)) has been investigated by a number of authors (see for example $[24,25]$ ) using beam elements as well as shell elements. The material properties are $E=71240$ (Young's modulus), $v=0.31$ (Poisson's ratio); the edge length is $l=240$, the edge width is $b=30$ and the thickness is $h=0.6$. The strip is subjected to an in-plane load in the free-edge. The post-buckling behaviour depends on the direction of the load which is indicated in the figure. A bifurcation point is arrived at using an extended system of equations [26]; a switch of the displacement path is performed at this point and the postbuckling path is followed using an arc-length method. Figure 7(b) shows the normalized post-critical path using a model with 258 elements (516 DOFs) for the present element $\left(\lambda_{\mathrm{cr}}=1.216\right)$. Results obtained using a transverse shear deformable quadratic triangle (34 elements and 510 DOFs) [27] are also plotted for reference $\left(\lambda_{\mathrm{cr}}=1.163\right)$. The 


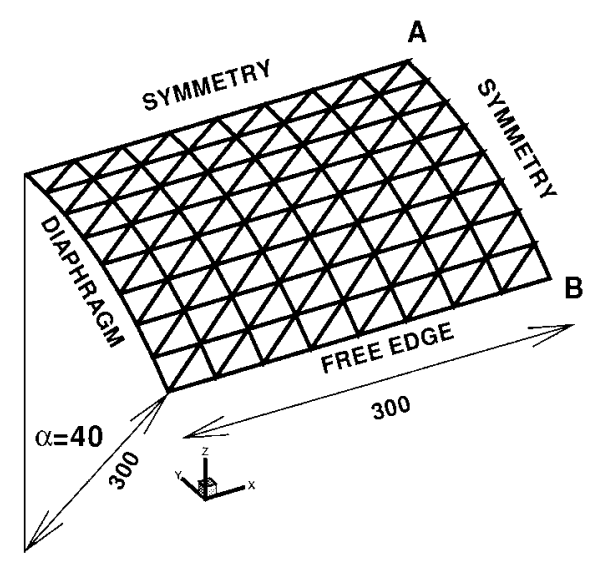

Figure 5. Cylindrical roof under uniform dead weight. $E=310^{6}, v=0.0$, thickness $=3.0$, shell weight $=0.625$ per unit area. (a)
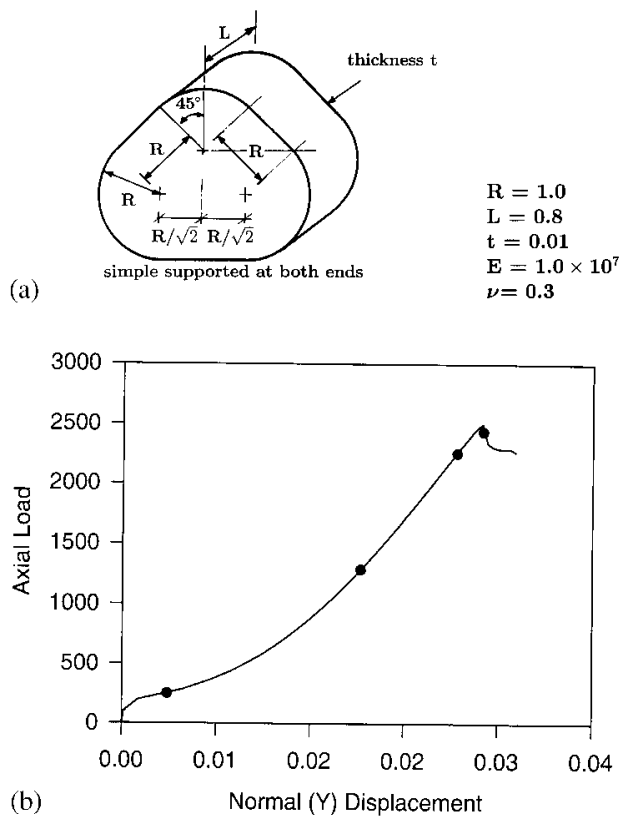

Figure 6. Pear-shaped cylinder: (a) geometry and material properties; and (b) displacements of point 1 vs. total axial load, ( - ) present element, $(\bullet)$ target values.

behaviour of the present element is a little stiffer than the quadratic triangle but has better convergence properties.

9.2.3. Pinched hemispherical shell. This example has also been presented as a benchmark in Reference [22] and analysed in Reference [25]. An hemispherical shell of radius $r=10$ and thickness $h=0.04$, with an $18^{\circ}$ hole at the pole and free at all boundaries is subjected to two inward and two outward forces $90^{\circ}$ apart. The material properties are $E=6.825 \times 10^{7}$ and $v=0.3$. Only one quadrant is modeled due to symmetry with 640 BST elements (1036 DOFs). Figure 8(a) shows the geometry and discretization used; the deformed geometry (without magnification) is also shown for $\lambda=100$. The displacements of the loaded points are plotted in Figure $8(\mathrm{~b})$ as a function of the load factor. The BST element results compare very well with both the target results obtained with ABAQUS [23] and the results from Reference [25].

9.2.4. Pinched cylinder with isotropic hardening. A short cylinder bounded by two rigid diaphragms at its ends, is subjected to opposite load at the middle section. Due to symmetry only one-eighth is discretized with 3528 BST elements involving 1849 nodes and 5336 DOFs. The geometry of the cylinder is: length $L=600$, radius $r=300$, thickness $t=3$. The material properties are: Young's modulus $E=3 \times 10^{3}$, Poisson's ratio $v=0.3$, initial yield stress $k_{0}=24.3$ and isotropic hardening modulus $k^{\prime}=30.0$. Figure 9(a) plots the applied load factor 
(a)
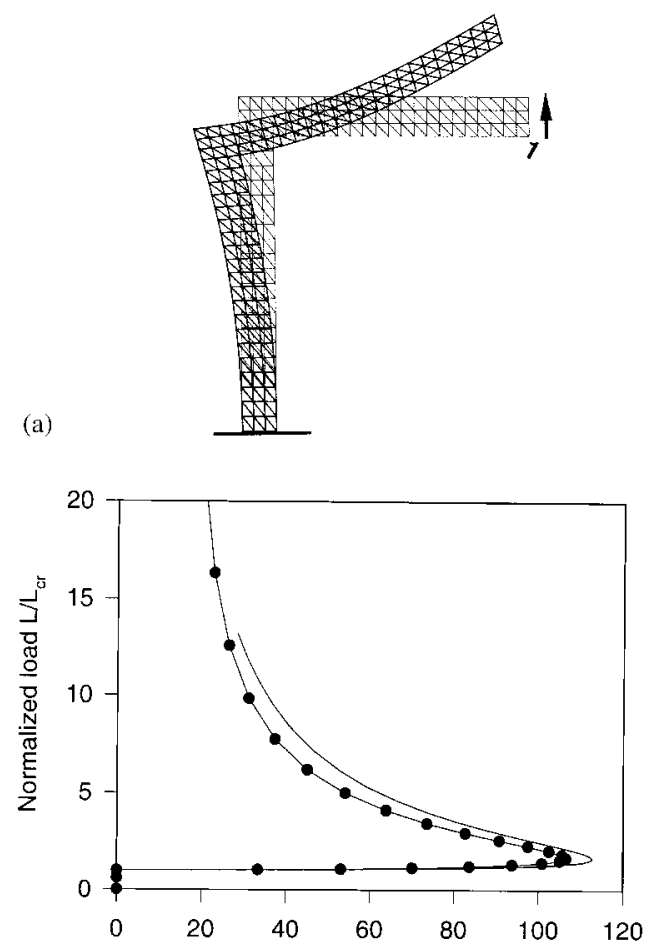

(b)

Normalized displacements w/h

Figure 7. L-shaped plate strip under in-plane loading: (a) geometry and deformed shape for $\lambda / \lambda_{\mathrm{cr}}=1.67$; (b) normalized displacements of point 1 vs. normalized load, $(-)$ quadratic triangle, $(\bullet)$ BST.

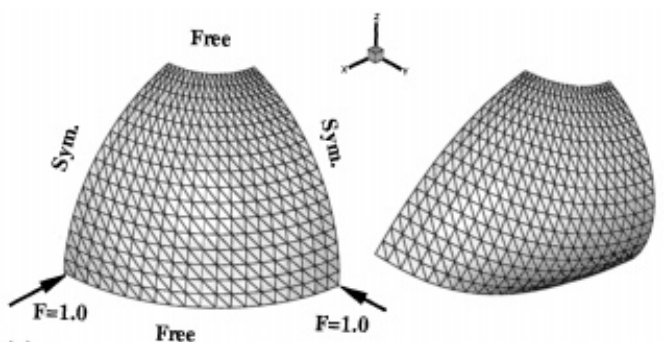

(a)

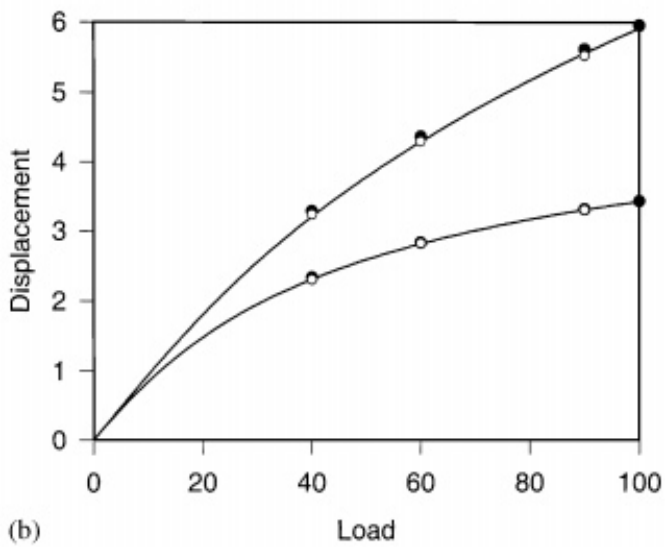

Figure 8. Pinched hemispherical shell: (a) geometry and deformed shape for $\lambda=100$; and; (b) displacements of loaded points vs. load factor; (- $)$ present element, (•) target results, (०) results of Ref. [23].

versus the load point displacements. Figure 9(b) shows the deformed configuration for a load displacement of 180 (60 per cent of the radius) and the effective plastic strain contours at the external face. Small strain kinematics were considered in this case to avoid an early collapse due to unbounded plastic strain growth under the loaded point. The numerical results obtained agree with those reported in References [25, 28].

9.2.5. Hemispherical Punch Stretching. This benchmark was proposed in Reference [29]. The geometry and material properties are shown in Figure 10(a). A friction coefficient of $\mu=0.3$ was adopted. A fine mesh of 800 BST elements was used to model one-quarter of the geometry due to symmetry. Figure 10(b) shows the total force over the punch as a function of the punch travel. Figure 10(c) shows the thickness distribution for different punch displacements. Both results are in excellent agreement with results obtained with a membrane formulation using logarithmic strains [30] as well as with a rigid-viscoplastic formulation [31]. 


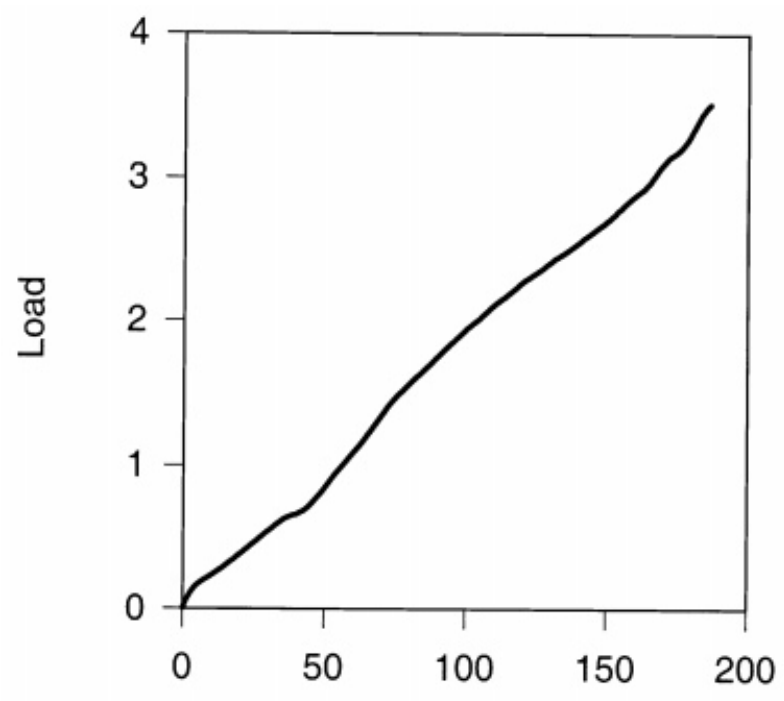

(a)

Displacement under the load

(b)

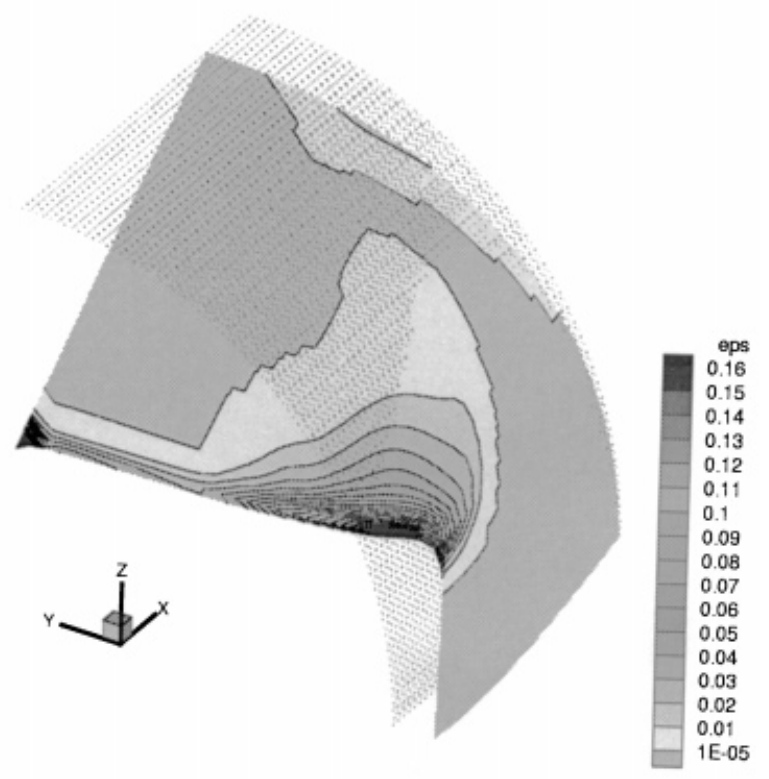

Figure 9. Pinched cylinder: (a) load factor vs. load displacement; and (b) deformed geometry and plastic strain contours.

9.2.6. Deep drawing of a square cup. This example reproduces one of the benchmarks proposed at NUMISHEET'93 [32]. It is the deep-drawing of a mild steel square cup. One-quarter of the geometry has been modelized due to symmetry conditions. Figure 11 shows the geometry definition of tools (matrix, punch, and blank-holder) and the blank. Figure 12(a) shows 


$$
\begin{aligned}
& E=69004 \mathrm{MPa} \\
& \nu=0.3 \\
& \sigma_{y}=589\left(10^{-4}+\bar{e}_{p}\right)^{0.216}
\end{aligned}
$$
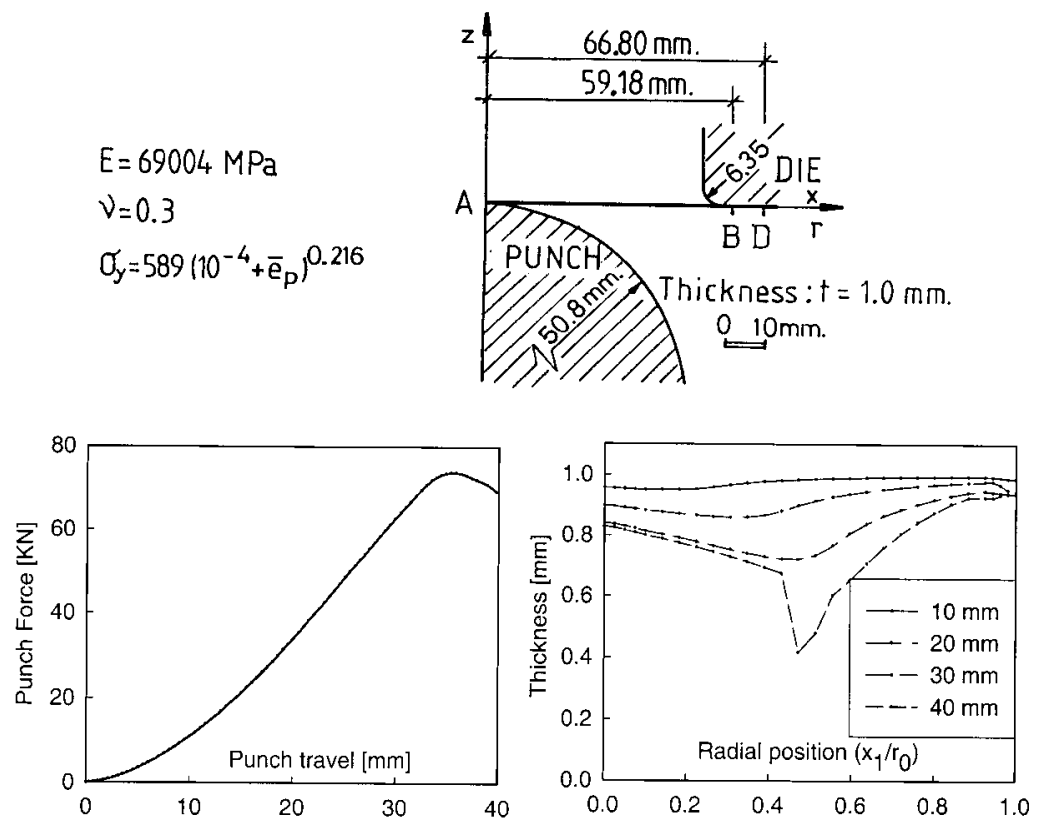

Figure 10. Hemispherical punch stretching.
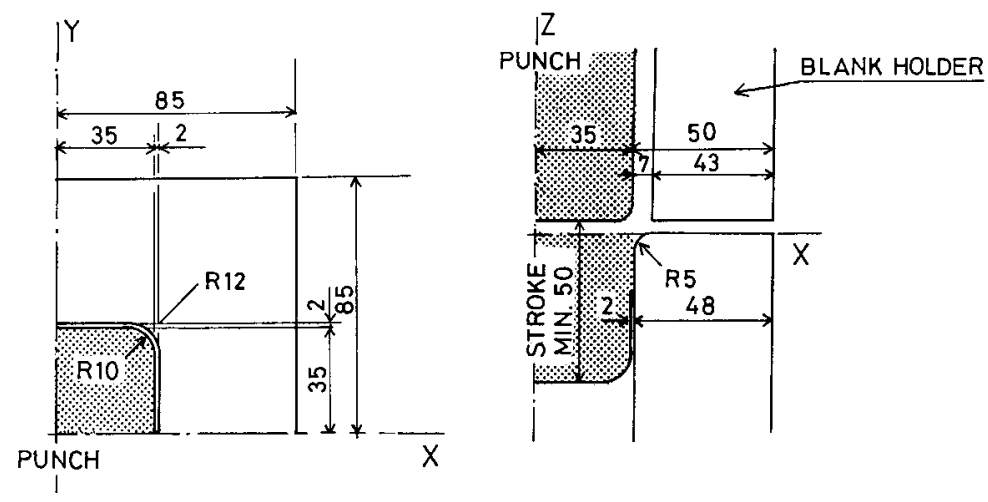

Figure 11. Deep-drawing of a square cup. Geometry definition.

the deformed mesh of the sheet after the final travel of the punch and contour lines of the logarithmic strain in the thickness direction. The final draw-in values are $D X=29.84 \mathrm{~mm}$ and $D D=15.95 \mathrm{~mm}$ that compare quite well with the average of the experimental values $(D X=27.95 \mathrm{~mm}$ and $D D=15.36 \mathrm{~mm})$. Figure 12(b) shows the logarithmic strain distribution along lines $\mathrm{OA}$ and $\mathrm{OB}$. Comparisons with the experimental values show the same differences encountered with other numerical simulations [32].

Other examples of the good behaviour of the BST element for sheet stamping analysis can be found in [15-17]. 

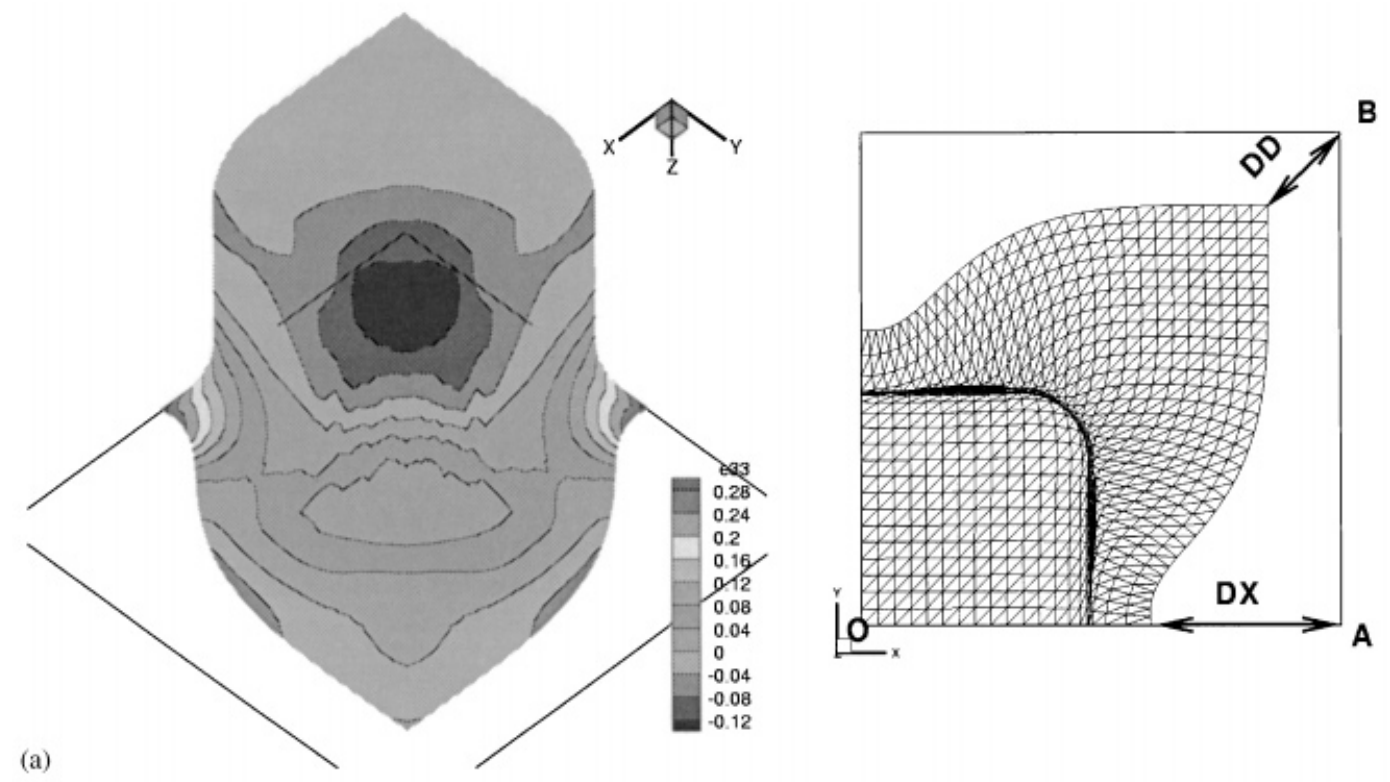

(a)
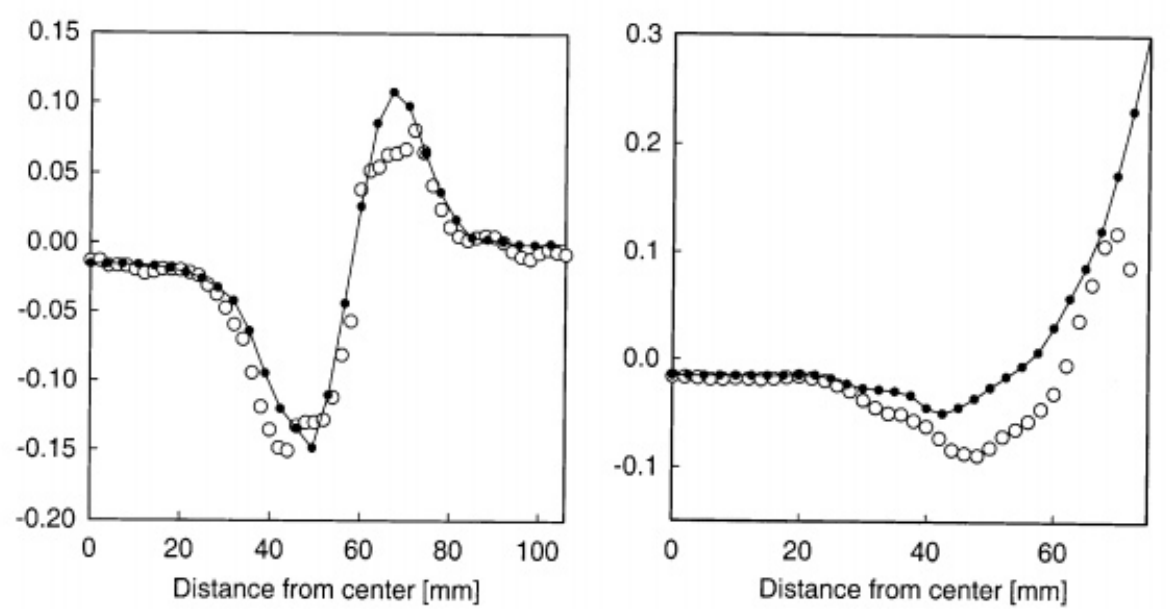

Figure 12. Deep-drawing of a square cup: (a) deformed shape and thickness contours; (b) logarithmic thickness strain along lines $O B$ and $O A$; and (०) average of experimental results; (•) BST element.

\section{CONCLUDING REMARKS}

A simple rotation-free shell triangle for large strain analysis of thin shells has been presented. The new BST element is based in a Total Lagrangian formulation with an hyper-elastic-based constitutive law that makes it adequate (objective) for implicit and explicit analysis.

The element has only translational DOFs, which makes it simple, but implies restrictions in the possibilities to mix the present element with other types of elements. Improvements in the 
treatment of simple supported and free edge boundary conditions are still possible. However, for the many examples solved, the results agree quite well with results from other references.

In the linear examples tested the element performance is better than that of standard triangular plate and shell elements measured in terms of the number of DOFs (for published results obtained with structured meshes). The performance, however, deteriorates slightly for unstructured meshes due to the way in which the curvatures are computed. Work to improve the element performance for very irregular meshes is in progress.

Very good results have also been obtained in examples including large displacements and large strains using implicit and explicit formulations.

In summary, the BST element shows many promising features for analysis of large-scale linear and non-linear shell problems.

\section{APPENDIX}

The computation of $\Delta\left(\delta \mathbf{t}_{3}\right)$ is derived here. Recall that

$$
\begin{aligned}
\delta \mathbf{t}_{3} & =-\left[\left(\mathbf{t}_{3} \cdot \delta \boldsymbol{\varphi}^{\prime} \alpha\right) \tilde{\boldsymbol{\varphi}}_{\prime_{\alpha}}\right] \\
& =-\left[\left(\tilde{\boldsymbol{\varphi}}_{\alpha} \otimes \mathbf{t}_{3}\right) \delta \boldsymbol{\varphi}^{\prime} \alpha\right]
\end{aligned}
$$

where a repeated greek index indicates summation $(\alpha=1,2)$

$$
\begin{aligned}
\Delta\left(\delta \mathbf{t}_{3}\right) & =-\left[\left(\Delta \tilde{\boldsymbol{\varphi}}_{\prime^{\alpha}} \otimes \mathbf{t}_{3}\right)+\left(\tilde{\boldsymbol{\varphi}}_{\alpha_{\alpha}} \otimes \Delta \mathbf{t}_{3}\right)\right] \delta \boldsymbol{\varphi}^{\prime \alpha} \\
& =\left[-\left(\Delta \tilde{\boldsymbol{\varphi}}_{\prime_{\alpha}} \otimes \mathbf{t}_{3}\right)+\left(\tilde{\boldsymbol{\varphi}}_{{ }^{\prime} \alpha} \otimes \tilde{\boldsymbol{\varphi}}_{\prime^{\prime}}\right)\left(\mathbf{t}_{3} \cdot \Delta \boldsymbol{\varphi}^{\prime} \beta\right)\right] \delta \boldsymbol{\varphi}^{\prime} \alpha
\end{aligned}
$$

Noting next that

$$
\begin{aligned}
\tilde{\boldsymbol{\varphi}}_{{ }_{\alpha}} \cdot \boldsymbol{\varphi}^{\prime} \beta & =\delta_{\alpha \beta} \\
\Delta \tilde{\boldsymbol{\varphi}}_{{ }_{\alpha}} \cdot \boldsymbol{\varphi}^{\prime} \beta & =-\tilde{\boldsymbol{\varphi}}_{{ }^{\alpha}} \cdot \Delta \boldsymbol{\varphi}^{\prime} \beta \\
\tilde{\boldsymbol{\varphi}}_{{ }_{\alpha}} \cdot \mathbf{t}_{3} & =0 \\
\Delta \tilde{\boldsymbol{\varphi}}_{{ }_{\alpha}} \cdot \mathbf{t}_{3} & =-\tilde{\boldsymbol{\varphi}}_{{ }_{\alpha}} \cdot \Delta \mathbf{t}_{3}
\end{aligned}
$$

and resolving $\Delta \tilde{\boldsymbol{\varphi}}_{{ }_{\alpha}}$ in the convective base

$$
\begin{aligned}
\Delta \tilde{\boldsymbol{\varphi}}_{{ }^{\alpha}} & =-\left[\left(\tilde{\boldsymbol{\varphi}}_{\alpha \alpha} \cdot \Delta \boldsymbol{\varphi}_{\beta}\right) \tilde{\boldsymbol{\varphi}}_{\beta}+\left(\tilde{\boldsymbol{\varphi}}_{\alpha} \cdot \Delta \mathbf{t}_{3}\right) \mathbf{t}_{3}\right] \\
& =-\left[\left(\tilde{\boldsymbol{\varphi}}_{\beta} \otimes \tilde{\boldsymbol{\varphi}}_{\alpha}\right) \Delta \boldsymbol{\varphi}_{\beta}+\left(\mathbf{t}_{3} \otimes \tilde{\boldsymbol{\varphi}}_{\alpha}\right) \Delta \mathbf{t}_{3}\right]
\end{aligned}
$$


one can write

$$
\begin{aligned}
& \Delta\left(\delta \mathbf{t}_{3}\right)=\left\{\left[\left(\tilde{\boldsymbol{\varphi}}_{{ }^{\alpha}} \cdot \Delta \boldsymbol{\varphi}^{\prime \beta}\right) \tilde{\boldsymbol{\varphi}}_{{ }^{\prime} \beta}+\left(\tilde{\boldsymbol{\varphi}}_{\alpha_{\alpha}} \cdot \Delta \mathbf{t}_{3}\right) \mathbf{t}_{3}\right] \otimes \mathbf{t}_{3}+\left(\tilde{\boldsymbol{\varphi}}_{\alpha_{\alpha}} \otimes \tilde{\boldsymbol{\varphi}}_{\prime^{\prime}}\right)\left(\mathbf{t}_{3} \cdot \Delta \boldsymbol{\varphi}^{\prime} \beta\right)\right\} \delta \boldsymbol{\varphi}^{\prime \alpha} \\
& =\left\{\left(\tilde{\boldsymbol{\varphi}}_{{ }_{\alpha}} \cdot \Delta \boldsymbol{\varphi}_{\prime} \beta\right)\left(\tilde{\boldsymbol{\varphi}}_{{ }^{\prime}} \otimes \mathbf{t}_{3}\right)+\left(\tilde{\boldsymbol{\varphi}}_{\alpha^{\prime}} \cdot \Delta \mathbf{t}_{3}\right)\left(\mathbf{t}_{3} \otimes \mathbf{t}_{3}\right)+\left(\mathbf{t}_{3} \cdot \Delta \boldsymbol{\varphi}_{\prime} \beta\right)\left(\tilde{\boldsymbol{\varphi}}_{\alpha_{\alpha}} \otimes \tilde{\boldsymbol{\varphi}}_{{ }^{\prime} \beta}\right)\right\} \delta \boldsymbol{\varphi}^{\prime \alpha}
\end{aligned}
$$

Replacing now the term $\Delta \mathbf{t}_{3}$ one has

$$
\begin{aligned}
& \Delta\left(\delta \mathbf{t}_{3}\right)=\left\{\left(\tilde{\boldsymbol{\varphi}}_{\prime_{\alpha}} \cdot \Delta \boldsymbol{\varphi}^{\prime} \beta\right)\left(\tilde{\boldsymbol{\varphi}}_{\prime \beta} \otimes \mathbf{t}_{3}\right)-\tilde{\boldsymbol{\varphi}}_{\prime_{\alpha}} \cdot\left[\left(\mathbf{t}_{3} \cdot \Delta \boldsymbol{\varphi}^{\prime} \beta\right) \tilde{\boldsymbol{\varphi}}_{\beta}\right]\left(\mathbf{t}_{3} \otimes \mathbf{t}_{3}\right)+\left(\mathbf{t}_{3} \cdot \Delta \boldsymbol{\varphi}^{\prime} \beta\right)\left(\tilde{\boldsymbol{\varphi}}_{\alpha_{\alpha}} \otimes \tilde{\boldsymbol{\varphi}}_{\prime^{\prime}}\right)\right\} \delta \boldsymbol{\varphi}^{\prime \alpha} \\
& =\left\{\left(\tilde{\boldsymbol{\varphi}}_{\alpha^{\alpha}} \cdot \Delta \boldsymbol{\varphi}^{\prime} \beta\right)\left(\tilde{\boldsymbol{\varphi}}_{\beta} \otimes \mathbf{t}_{3}\right)-\left(\tilde{\boldsymbol{\varphi}}_{\alpha^{\prime}} \cdot \tilde{\boldsymbol{\varphi}}_{{ }^{\prime} \beta}\right)\left(\mathbf{t}_{3} \cdot \Delta \boldsymbol{\varphi}^{\prime} \beta\right)\left(\mathbf{t}_{3} \otimes \mathbf{t}_{3}\right)+\left(\mathbf{t}_{3} \cdot \Delta \boldsymbol{\varphi}^{\prime} \beta\right)\left(\tilde{\boldsymbol{\varphi}}_{{ }^{\prime} \alpha} \otimes \tilde{\boldsymbol{\varphi}}_{\beta}\right)\right\} \delta \boldsymbol{\varphi}^{\prime} \alpha
\end{aligned}
$$

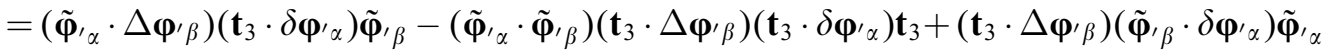

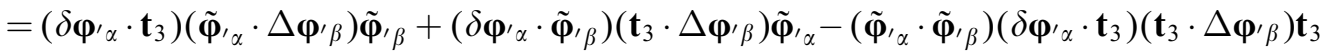

$$
\begin{aligned}
& =\left[\delta \boldsymbol{\varphi}_{\prime_{\alpha}}^{T}\left(\mathbf{t}_{3} \otimes \tilde{\boldsymbol{\varphi}}_{\alpha \alpha}\right) \Delta \boldsymbol{\varphi}^{\prime} \beta\right] \tilde{\boldsymbol{\varphi}}_{{ }^{\prime} \beta}+\left[\delta \boldsymbol{\varphi}_{\prime_{\alpha}}^{T}\left(\tilde{\boldsymbol{\varphi}}_{{ }^{\prime} \beta} \otimes \mathbf{t}_{3}\right) \Delta \boldsymbol{\varphi}^{\prime} \beta\right] \tilde{\boldsymbol{\varphi}}_{\alpha^{\alpha}}-\left(\tilde{\boldsymbol{\varphi}}_{\alpha^{\alpha}} \cdot \tilde{\boldsymbol{\varphi}}_{\prime^{\prime}}\right)\left[\delta \boldsymbol{\varphi}_{\prime_{\alpha}}^{T}\left(\mathbf{t}_{3} \otimes \mathbf{t}_{3}\right) \Delta \boldsymbol{\varphi}^{\prime} \beta\right] \mathbf{t}_{3}
\end{aligned}
$$

\section{ACKNOWLEDGEMENTS}

The first author is a member of the scientific staff of the Science Research Council of Argentina (CONICET). The support provided by grants of CONICET and CONICOR is gratefully acknowledged.

\section{REFERENCES}

1. Nay RA, Utku S. An alternative to the finite element method. Variational Methods Engineering 1972; 1(3): 62-74.

2. Barnes MR. Form finding and analysis of tension space structure by dynamic relaxation, Ph.D. Thesis, Department of Civil Engineering, The City University, London, 1977.

3. Hampshire JK, Topping BHV, Chan HC. Three node triangular elements with one degree of freedom per node. Engineering and Computation 1992; 9:49-62.

4. Phaal R, Calladine CR. A simple class of finite elements for plate and shell problems. I: elements for beams and thin plates. International Journal for Numerical Methods in Engineering 1992; 35:955-977.

5. Phaal R, Calladine CR. A simple class of finite elements for plate and shell problems. II: an element for thin shells with only translational degrees of freedom. International Journal for Numerical Methods in Engineering 1992; 35: 979-996.

6. Oñate E. A review of some finite element families for thick and thin plate and shell analysis. In Recent Development in Finite Element Analysis, Hughes TJR, Oñate E, Zienkiewicz OC (eds). CIMNE: Barcelona, 1999.

7. Yang DY, Jung DW, Song LS, Yoo DJ, Lee JH. Comparative investigation into implicit, explicit and iterative implicit/explicit schemes for simulation of sheet metal forming processes. NUMISHEET'93, Makinouchi A, Nakamachi E, Oñate E, Wagoner RH (eds). RIKEN: Tokyo, 1993; 35-42.

8. Brunet M, Sabourin F. Prediction of necking and wrinkles with a simplified shell element in sheet forming. In: Kröplin B (ed.), International Conference of Metal Forming Simulation in Industry, vol. II, 1994; 27-48.

9. Rio G, Tathi B, Laurent H. A new efficient finite element model of shell with only three degrees of freedom per node. Applications to industrial deep drawing test. In Recent Developments in Sheet Metal Forming Technology, Barata Marques MJM (ed.), 18th IDDRG Biennial Congress, Lisbon, 1994.

10. Oñate E, Cervera M. Derivation of thin plate bending elements with one degree of freedom per node. Engineering and Computation 1993; 10:543-561.

11. Oñate E, Cervera M, Zienkiewicz OC. A finite volume format for structural mechanics. International Journal for Numerical Methods in Engineering, 1994; 37:181-201. 
12. Zienkiewicz OC, Oñate E. Finite elements vs. finite volumes. Is there really a choice? Nonlinear Computational Mechanics. State of the Art, Wriggers P, Wagner R (eds). Springer: Heidelberg, 1991.

13. Zárate F. New finite elements for plate and shell analysis. Ph.D. Thesis, Univ. Politècnica de Catalunya, Barcelona, 1996 (in Spanish).

14. Oñate E, Zárate F. Rotation-free plate and shell triangles. International Journal for Numerical Methods in Engineering, 2000; 47:557-603.

15. Cendoya P. Explicit dynamic analysis of shells using rotational dof-free triangles. Ph.D. Thesis, Univ. Politècnica de Catalunya, Barcelona, 1996 (in Spanish).

16. Jovicevic J, Oñate E. Analysis of beam and shells using a rotation-free finite element-finite volume formulation, Monograph CIMNE, International Center for Numerical Methods in Engineering, Barcelona, 1999.

17. Oñate E, Zárate F, Rojek J, Duffet G, Neamtu L. Advances in rotation-free shell elements for sheet stamping analysis. NUMISHEET'99, Gelin JC, Picart P (eds). BURS Edition: Besan, 1999.

18. Eterovic A. A study of formulations for large strain elasto-plastic finite element analysis. M.Sc. Thesis, MIT: Cambridge, USA, 1989.

19. Belytschko T, Stolarski H, Carpenter N. A $\mathrm{C}^{0}$ triangular plate element with one point quadrature. International Journal for Numerical Methods in Engineering 1984; 20:787-802.

20. Huang HC. Static and Dynamic Analysis of Plates and Shells. Springer: Berlin, 1989; 40.

21. Carpenter N, Stolarski H, Belytschko T. Improvements in 3-node triangular shell elements. International Journal for Numerical Methods in Engineering 1986; 23:1643-1667.

22. Prinja NK, Clegg RA. A review of benchmark problems for geometric non-linear behavior of 3-D beams and shell. NAFEMS, September 1993.

23. Karlson H, Sorensen Inc. ABAQUS, version 5.2.1, Pawtucket, USA.

24. Argyris JH, Balmer H, Doltsinis J St, Dunne PC, Hasse M, Kleiber M, Malejannakis GA, Mlejenek HP, Muller M, Scharpf DW. Finite element method - the natural approach. Computer Methods in Applied Mechanics and Engineering 1979; 17/18:1-106.

25. Simo JC, Fox DD, Rifai MS. On a stress resultant geometrically exact shell model. Part III: computational aspects of the non-linear theory. Computer Methods in Applied Mechanics and Engineering 1990; 79:21-70.

26. Wriggers P, Simo JC. A general procedure for the direct computation of turning and bifurcation points. International Journal for Numerical Methods in Engineering 1990; 30:155-176.

27. Flores FG, Oñate E, Zárate F. New assumed strain triangles for non-linear shell analysis. Computational Mechanics 1995; 17:107-114.

28. Hauptmann R, Schweizerhof K. A systematic development of 'solid-shell' element formulations for linear and non-linear analyses employing only displacement degrees of freedom. International Journal for Numerical Methods in Engineering 1998; 42:46-69.

29. Lee JK, Wagoner RH, Nakamachi E. A benchmark test for sheet metal forming. Technical Report, Ohio State University, 1990.

30. de Souza Neto EA, Peric D, Owen R. Continuing modelling and numerical simulation of material damage at finite strains. Archives of Computer Methods in Engineering 1998; 5(4):311-384.

31. Oñate E, Agelet de Saracibar C. Finite element analysis of sheet forming problems using a selective viscous bending/membrane formulation. International Journal for Numerical Methods in Engineering 1990; 30: $1577-1593$

32. Makinouchi A, Nakamachi E, Oñate E, Wagoner R. (eds). Proceedings of the International Conference NUMISHEET'93, Riken, Tokyo, 1993. 